\title{
KECK/NIRC2 IMAGING OF THE WARPED, ASYMMETRIC DEBRIS DISK AROUND HD 32297
}

\author{
Thayne Currie ${ }^{1}$, Timothy J. Rodigas ${ }^{2}$, John Debes ${ }^{3}$, Peter Plavchan ${ }^{4}$, Marc Kuchner $^{1}$, Hannah Jang-Condell ${ }^{5}$,

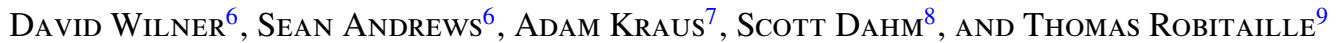 \\ ${ }^{1}$ NASA-Goddard Space Flight Center, Greenbelt, MD 20771, USA \\ ${ }^{2}$ Steward Observatory, University of Arizona, 933 North Cherry Avenue, Tucson, AZ 85721, USA \\ ${ }^{3}$ Space Telescope Science Institute, 3700 San Martin Drive, Baltimore, MD 21218, USA \\ ${ }^{4}$ NEXSCI, California Institute of Technology, Pasadena, CA 91125, USA \\ ${ }^{5}$ Department of Physics and Astronomy, University of Wyoming, Laramie, WY 82071, USA \\ ${ }^{6}$ Harvard-Smithsonian Center for Astrophysics, 60 Garden Street, Cambridge, MA 02138, USA \\ ${ }^{7}$ Institute for Astronomy, University of Hawaii, 2680 Woodlawn Drive, Honolulu, HI 96822, USA \\ ${ }^{8}$ Keck Observatory, 65-1120 Mamalahoa Highway, HI 96743, USA \\ ${ }^{9}$ MPIA-Heidelberg, Königstuhl 17, D-69117 Heidelberg, Germany \\ Received 2012 May 5; accepted 2012 July 17; published 2012 August 31
}

\begin{abstract}
We present Keck/NIRC2 $K_{s}$-band high-contrast coronagraphic imaging of the luminous debris disk around the nearby, young A star HD 32297 resolved at a projected separation of $r=0^{\prime \prime} .3-2$.'5 ( $\approx 35-280$ AU). The disk is highly warped to the north and exhibits a complex, "wavy" surface brightness (SB) profile interior to $r \approx 110$ AU, where the peaks/plateaus in the profiles are shifted between the NE and SW disk lobes. The SW side of the disk is 50\%-100\% brighter at $r=35-80 \mathrm{AU}$, and the location of its peak brightness roughly coincides with the disk's millimeter (mm) emission peak. Spectral energy distribution modeling suggests that HD 32297 has at least two dust populations that may originate from two separate belts, likely at different locations, possibly at distances coinciding with the SB peaks. A disk model for a single dust belt including a phase function with two components and a 5-10 AU pericenter offset explains the disk's warped structure and reproduces some of the SB profile's shape (e.g., the overall "wavy" profile, the SB peak/plateau shifts) but more poorly reproduces the disk's brightness asymmetry and the profile at wider separations $(r>110 \mathrm{AU})$. Although there may be alternate explanations, agreement between the SW disk brightness peak and disk's peak mm emission is consistent with an overdensity of very small, sub-blowout-sized dust and large, $0.1-1 \mathrm{~mm}$ sized grains at $\approx 45 \mathrm{AU}$ tracing the same parent population of planetesimals. New near-IR and submillimeter observations may be able to clarify whether even more complex grain scattering properties or dynamical sculpting by an unseen planet are required to explain HD 32297's disk structure.
\end{abstract}

Key words: planetary systems - stars: early-type - stars: individual (HD 32297)

Online-only material: color figures

\section{INTRODUCTION}

Debris disks are signposts of planets and planet formation (e.g., Wyatt 2008; Kenyon \& Bromley 2008). Supporting this picture, the two stars with independently confirmed, directly imaged planetary systems, HR 8799 and $\beta$ Pictoris (Marois et al. 2008, 2010; Currie et al. 2011a; Lagrange et al. 2010), are surrounded by luminous debris disks (Smith \& Terrile 1984; Rhee et al. 2007; Su et al. 2009). Similarly, Fomalhaut has a candidate planetary companion located just interior to the star's bright debris ring (Kalas et al. 2008).

In the absence of a directly imaged planet, resolved imaging of debris disks may provide indirect evidence for a massive planet's existence, may help constrain the unseen planet's properties, and thus can help identify promising targets for future direct imaging (e.g., Wyatt et al. 1999). For example, the inclined or "warped" component of $\beta$ Pictoris's debris disk (Heap et al. 2000; Golimowski et al. 2006) is likely due to the directly imaged planet (Augereau et al. 2001; Dawson et al. 2011) and also provides an estimate for the planet's mass independent of planet cooling models (Lagrange et al. 2010). Dynamical sculpting by a planet/planets may explain the sharp inner edge and pericenter offset of Fomalhaut's debris ring (Kalas et al. 2005, 2008; Quillen et al. 2006). Other debris disk structures may be due to non-planet processes, in particular interactions with the interstellar medium (ISM) or perturbations from a nearby star, as has been proposed to explain images of disks around HD 15115 and HD 61005 (e.g., Kalas et al. 2007; Hines et al. 2007).

The nearby $\left(d=112_{-10}^{+12} \mathrm{pc}\right.$; van Leeuwen 2007) A5 star HD 32297 is another example of a young star surrounded by a luminous, spatially resolved debris disk. At $30 \mathrm{Myr}$ old (Kalas et al. 2005), it is roughly coeval with HR 8799 and may probe debris disk evolution at a stage just after they are most collisionally active (Kenyon \& Bromley 2008; Currie et al. 2008, 2009). Like $\beta$ Pic and HR 8799, HD 32297 has a large infrared (IR) excess emission due to circumstellar dust first identified from IRAS data. Schneider et al. (2005) thus selected HD 32297 for Hubble Space Telescope NICMOS (F110W) coronagraphic imaging and resolved the disk out to an angular distance (from the star) of 3".3 ( 400 AU). HD 32297 was subsequently resolved in the optical (Kalas 2005), near-IR (1.6-2.2 $\mu \mathrm{m}$; Debes et al. 2009; Mawet et al. 2009), thermal infrared (10-20 $\mu \mathrm{m}$; Moerchen et al. 2007; Fitzgerald et al. 2007), and millimeter (1.3 mm; Maness et al. 2008).

Previous work has claimed that HD 32297's disk structure is shaped by planet sculpting as well as non-planet processes. Debes et al. (2009) identified an asymmetry in the disk scattering efficiency between the northeast and southwest sides (see also Kalas 2005). They argued that ISM sculpting explains this 
feature much like it explains some properties of the HD 15115 and HD 61005 disks. Schneider et al. (2005) identified a brightness asymmetry between the two disk sides, a feature consistent with sculpting by a massive planet (see also Maness et al. 2008).

The two mechanisms, ISM sculpting and planets, are not mutually exclusive. New images of the HD 15115 and HD 61005 disks reveal cleared inner regions and/or pericenter offsets, both of which are plausibly due to a planetary companion (Rodigas et al. 2012; Buenzli et al. 2010). Additionally, multiple debris belts, scaled analogues to the solar system's asteroid belt and Kuiper Belt, are also likely planet signposts and may reside around HD 61005, HD 15115, and HD 32297 (Fitzgerald et al. 2010; Maness et al. 2008; Rodigas et al. 2012).

To determine which mechanisms are responsible for shaping HD 32297's debris disk structure, we need new, high signal-tonoise $(\mathrm{S} / \mathrm{N})$ images with which to derive precise disk properties. Although Schneider et al. (2005) identify a disk brightness asymmetry consistent with planet sculpting, they caution that the disk brightness measurements close to the coronagraphic spot $\left(r \sim 00^{\prime \prime} 3-00^{\prime \prime} 4\right)$ which provide the basis for this asymmetry are highly uncertain. Point-spread function (PSF) subtraction errors due to the completely opaque NICMOS coronagraphic spot may limit our ability to conclusively identify disk structure at these small, speckle-dominated separations. Moreover, if the asymmetry identified a planet-induced density structure, then it should align with the millimeter $(\mathrm{mm})$ emission peak (Maness et al. 2008). However, it is not clear whether these asymmetries are aligned and thus whether they identify small and large grains originating from the same parent population. The Palomar $/ K_{s}$ image from Mawet et al. (2009) has limited spatial resolution. While they did recover Schneider et al.'s brightness asymmetry, higher spatial resolution observations could confirm and help clarify the physical origin of this and other features. For example, new data could identify breaks in the disk brightness profile that may reveal evidence for the multiple debris belts inferred from modeling unresolved IR data.

To further clarify the nature of the HD 32297 debris disk, we present new coronagraphic imaging obtained at $K_{s}(\sim 2 \mu \mathrm{m})$ with the Keck telescope on Mauna Kea, resolving the disk at angular separations of $0.3-2$.'5. Section 2 describes our observations and extraction of the disk images. In Section 3, we investigate basic disk properties (position angle, full width half-maximum (FWHM), and surface brightness (SB)) as a function of angular separation from the star. We then combine imaging with new, unresolved broadband photometry from the Spitzer Space Telescope and the WISE satellite to constrain the disk structure and identify the location(s) of the disk emission (Section 4). Finally, we compare our analyses to those from previous work on HD 32297 (Section 5) and investigate the physical mechanisms responsible for sculpting the disk emission (Section 6).

\section{NIRC2 DATA}

\subsection{Observations}

We imaged HD 32297 on UT 2011 November 20 with the NIRC2 camera mounted on Keck II using the $K_{s}$ filter $\left(\lambda_{o}=\right.$ $2.16 \mu \mathrm{m})$ in the narrow camera mode $\left(9.952 \mathrm{mas}_{\mathrm{pixel}}{ }^{-1}\right.$; Yelda et al. 2010) with correlated double sampling. The Keck adaptive optics (AO) system delivered diffraction limited images with an FWHM of $\sim 4.9$ pixels ( $\sim 49$ mas). To enhance our ability to extract the HD 32297 disk emission from the bright stellar halo, we centered the star behind the 0.'6 diameter, partially transmissive coronagraphic spot and used the "large hex" pupil plane mask.

Our HD 32297 data consist of co-added 30 s exposures with a cumulative integration time of 20 minutes and were taken through transit $(\mathrm{HA}=[-0.22,0.35])$ in "vertical angle" or angular differential imaging mode (ADI; Marois et al. 2006). Over the course of these exposures, the parallactic angle changed by $36 \mathrm{deg}$. While light cirrus caused fluctuations in the source transmission (as measured from the PSF core behind the coronagraphic mask) on the order of $\sim 10 \%$, observing conditions were otherwise stable.

To flux calibrate the data, we measure the flux of the PSF core of HD 32297 as viewed through the partially transmissive coronagraphic mask and corrected for the extinction through the mask. To determine the extinction, we use Keck/NIRC2 $K_{s}$ observations of HD 15115 taken in 2011 August in photometric conditions with the same coronagraphic spot size that were fluxcalibrated with a standard star HD 3029. From the August data, we measure the extinction through the $0^{\prime \prime} 6$ spot to be $6.91 \pm$ 0.15 mag, slightly less than but comparable to extinction estimates in $K_{s}$ for $1^{\prime \prime}-2^{\prime \prime}$ spot diameters (Metchev et al. 2009). Uncertainties in the aperture correction inside the coronagraphic spot due to scattered light from the spot edge limit the precision of our estimate. We did observe a photometric standard (p486r) 90 minutes after our HD 32297 imaging sequence. Although conditions became highly variable during the standard star observations due to patchy cloud coverage, our absolute flux calibration implied from the least extincted standard star frames agrees with that derived from the coronagraph transmission to within $0.3 \mathrm{mag}$.

\subsection{Image Processing}

Basic image processing and high-contrast imaging techniques follow methods outlined in Currie et al. (2010, 2011a, 2011b). We employ standard dark subtraction and flat-fielding corrections, identify and interpolate over hot/cold pixels, and apply the distortion correction determined from Yelda et al. (2010). For image registration, we exploit the fact that the PSF core is visible through the coronagraphic mask and is unsaturated. We register each image to subpixel ( $\sigma_{\text {cen }} \approx 0.1$ pixels) accuracy by determining the centroid position of the first image in the sequence and then determining the relative offset between the reference image and subsequent images by solving for the peak in the cross-correlation function for each image pair. Finally, we subtract off a radial profile to remove the smooth seeing halo and make a second pass through the images to identify remaining bad pixels.

To extract a detection of the HD 32297 disk, we adopt the Locally Optimized Combination of Images approach (LOCI; Lafreniere et al. 2007), using an updated version of the LOCI-based code employed in Currie et al. (2011b), which will be detailed later (Currie et al. 2012; T. Currie 2012, in preparation). Following Thalmann et al. (2011), we reduce the data using "conservative" LOCI settings more appropriate for extended sources (i.e., disks, not planets), where we consider rotation gaps $\delta$ of $1.5-5 \times$ FWHM, optimization areas $\left(N_{A}\right)$ of $1000-3000 \times$ the FWHM area, optimization geometries $g$ of $0.5-2$, and subtraction annuli $(d r)$ of 5-10 pixels wide (see Lafreniere et al. 2007, for more details). To determine the $\mathrm{S} / \mathrm{N}$ per resolution element of our disk detection, we convolve the image with a beam size equal to $\sim 1$ FWHM and compare pixel counts to the standard deviation of counts within a 
1 FWHM-wide annulus. ${ }^{10}$ Finally, we correct for photometric/ astrometric biases inherent in LOCI-based processing by imputing fake disks into each registered image and comparing the predicted and measured disk properties (e.g., SB, FWHM, position angle). Our method follows that first developed by Rodigas et al. (2012) and is described in full in the Appendix.

\subsection{HD 32297 Disk Image}

Figure 1 shows the image and S/N map for our "conservative" LOCI reduction assuming a rotation gap criterion of $\delta \geqslant 2.5$, $N_{A}=3000, g=1$, and $d r=10$, which balances our ability to detect the disk at small separations by attenuating speckles (favoring smaller $\delta, N_{A}$ ) but without oversubtracting the disk (favoring larger $\delta, N_{A}$ ). As evidenced by the $\mathrm{S} / \mathrm{N}$ map, we detect the disk at $\mathrm{S} / \mathrm{N} \geqslant 3$ from $r=2$ ". 5 all the way to the edge of the coronagraphic spot at $0^{\prime \prime} 3$. The peak $\mathrm{S} / \mathrm{N}$ per pixel is $\sim 19$. The disk emission along the midplane is more than $10 \sigma$ significant at 0 .'85-1". 4, while some regions of disk emission at 0.'3-0.'5 on the SW side are still more than $5 \sigma$ significant. Visually inspecting the image and $\mathrm{S} / \mathrm{N}$ map reveals significant disk structure. Most notably, the disk emission traces a distinct "bow" shape, where the disk position angle clearly changes with radial separation.

Furthermore, the disk exhibits a significant brightness asymmetry at small separations $(r<0$ '.7). Figure 2 redisplays our disk image with a different color stretch to better illustrate the brightness differences between the two sides of the disk. Interior to $r=0$ '.35 (identified with a circle), the SW side is significantly brighter than both the NE side as well as any pixel value for residual speckles located at different azimuthal separations; the NE side has a peak brightness only slightly larger than the brightest speckle. Exterior to this separation, there are no residual speckles as bright as either side of the disk, and the $\mathrm{SW}$ is still clearly brighter at least out to $r \approx 0^{\prime \prime} .6$ (yellow/red region on the $\mathrm{SW}$ side).

Our Keck $K_{s}$ image agrees well with the previous best $K_{s}$ results, which were obtained with the extreme-AO wellcorrected sub-aperture (WCS) on Palomar presented by Mawet et al. (2009) using classical (not ADI) imaging. Convolved to the Palomar/WCS beam size (Figure 2, bottom panel), our image strongly resembles that of Mawet et al.'s. The disk appears highly asymmetric with the SW side being brighter extending all the way to the coronagraphic spot ( $0{ }^{\prime} .3$ in our image compared to $0{ }^{\prime \prime} 4$ in theirs). As with the Mawet et al. (2009) data, the NE side appears fainter and truncated. Moreover, the brightest portion of the disk on the SW side roughly overlaps with the mm continuum peak (Maness et al. 2008).

\section{ANALYSIS}

Here, we investigate the HD 32297 disk geometry and SB profile, following methods similar to those described in Rodigas et al. (2012). We perform analysis on the image shown in Figure 1. The disk properties we report are corrected for photometric/astrometric biases inherent in LOCI processing (see the Appendix).

\subsection{Disk FWHM}

To better assess the HD 32297 disk morphology, we measure the disk FWHM perpendicular to the disk's major axis as a

\footnotetext{
10 The focus of this paper is imaging and characterizing the HD 32297 debris disk. While we do not present data reductions with methods more optimized for point-source detection, we plan to do so in a future work.
}
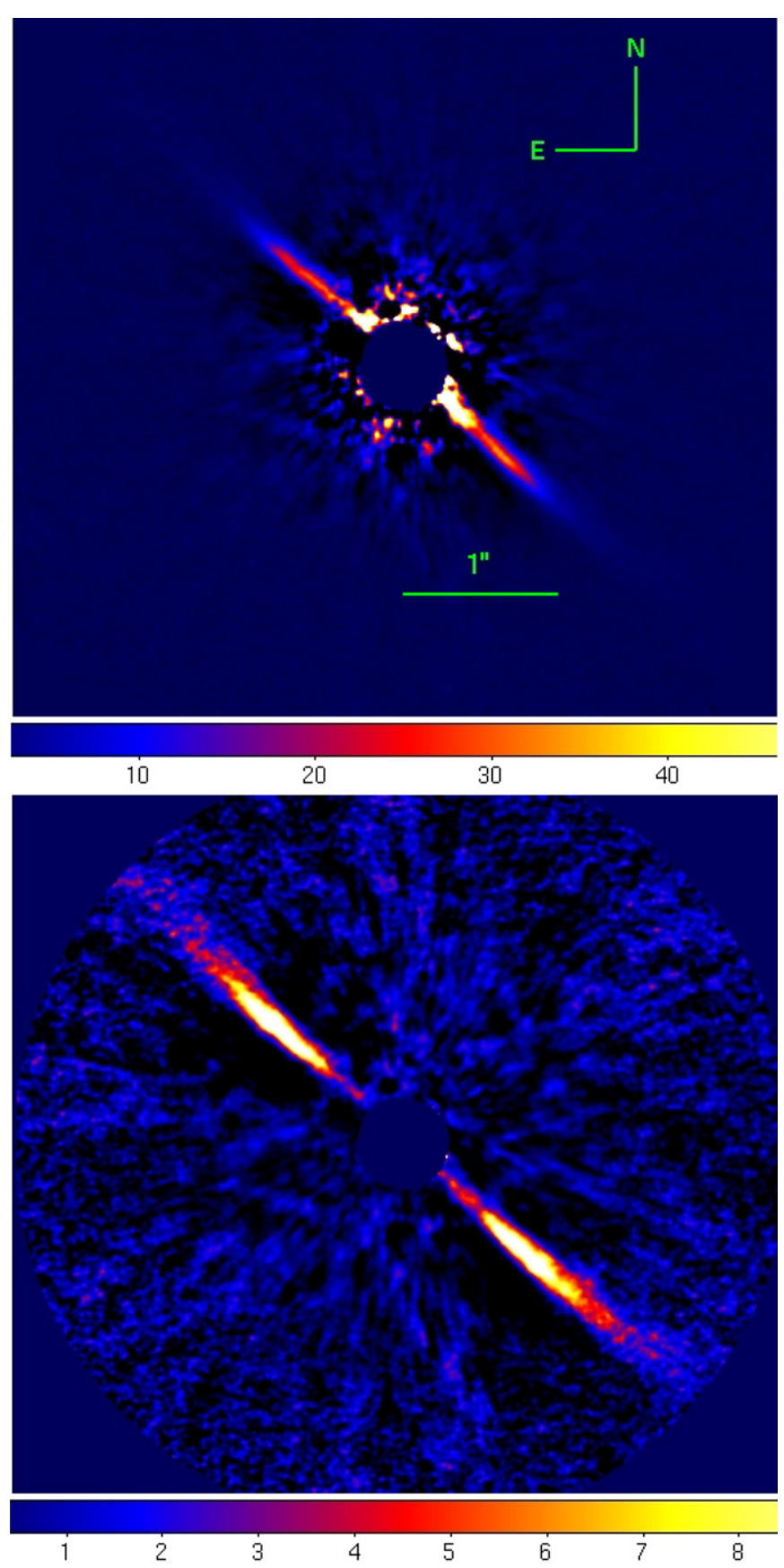

Figure 1. Reduced image (top) and signal-to-noise map (bottom) for our NIRC2 HD 32297 data. The color bar depicting units for the image are in counts, whereas they range from 0 to $9 \sigma$ for the signal-to-noise map. The central dark region identifies the coronagraphic spot $\left(r=0^{\prime \prime} .3\right)$. The panels have the same size scale. (A color version of this figure is available in the online journal.)

function of stellocentric distance. First, we identify the brightest pixels at each radial separation for the NE and SW lobes, respectively. Next, we place a 5 pixel by 21 pixel box centered on the brightest pixel and sum up the counts/pixel along each row of the box, producing a one-dimensional array of 21 values. Finally, we fit a Gaussian to this array, which yields the disk midplane location and the disk FWHM at that location.

Figure 3 displays the disk FWHM as a function of stellocentric distance for the NE (purple) and SW (green) sides. The errors correspond to the residuals of the Gaussian fits divided 

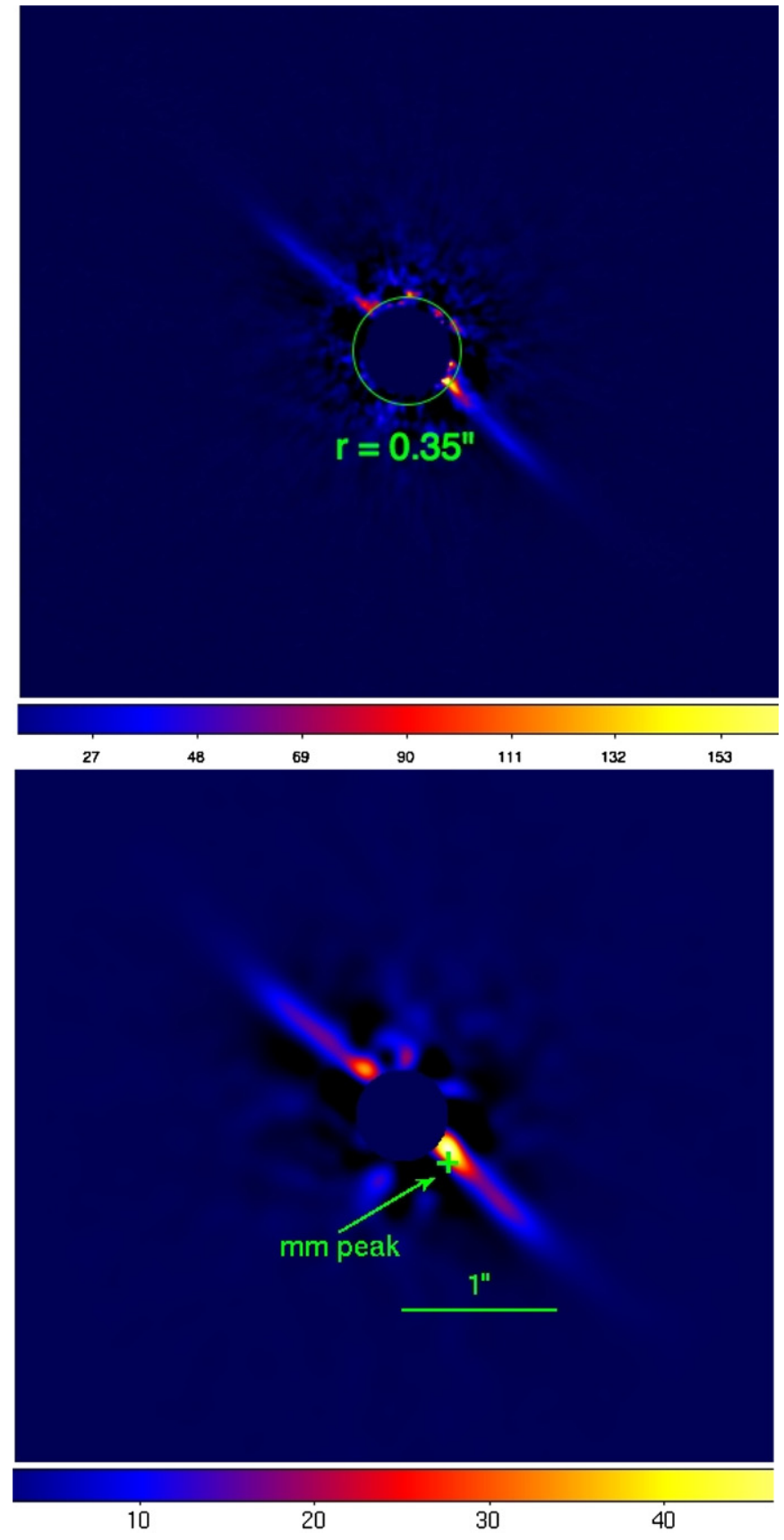

Figure 2. Top: our image from the top panel of Figure 1 redisplayed with a different color stretch to better illustrate the significance of our disk detection at the smallest separations ( $\left.r=0.0^{\prime} 3-0{ }^{\prime} 6\right)$ and the brightness asymmetry. Bottom: our image resampled to the same spatial resolution as the Palomar/WCS image from Mawet et al. (2009). The green cross identifies the position (and positiona uncertainties) of the peak brightness in the millimeter (Maness et al. 2008). Both panels are displayed in units of counts.

(A color version of this figure is available in the online journal.)

by the "throughput" for the disk FWHM as determined in the Appendix. On both sides, the disk FWHM steadily decreases from $\sim 0$ '. 25 at $r=1^{\prime \prime} .5$ to $\approx 0^{\prime}$. 15 at $r=1^{\prime \prime}$. Interior to $r=$ $1^{\prime \prime}$, the disk FWHM fluctuates about a constant value, though this behavior is likely due to the difficulty of fitting a Gaussian profile in speckle-dominated regions, not a bona fide clumpy structure. Beyond $r=1^{\prime \prime} .5$, where the disk emission approaches the photon noise limit, the FWHM estimates fluctuate wildly.
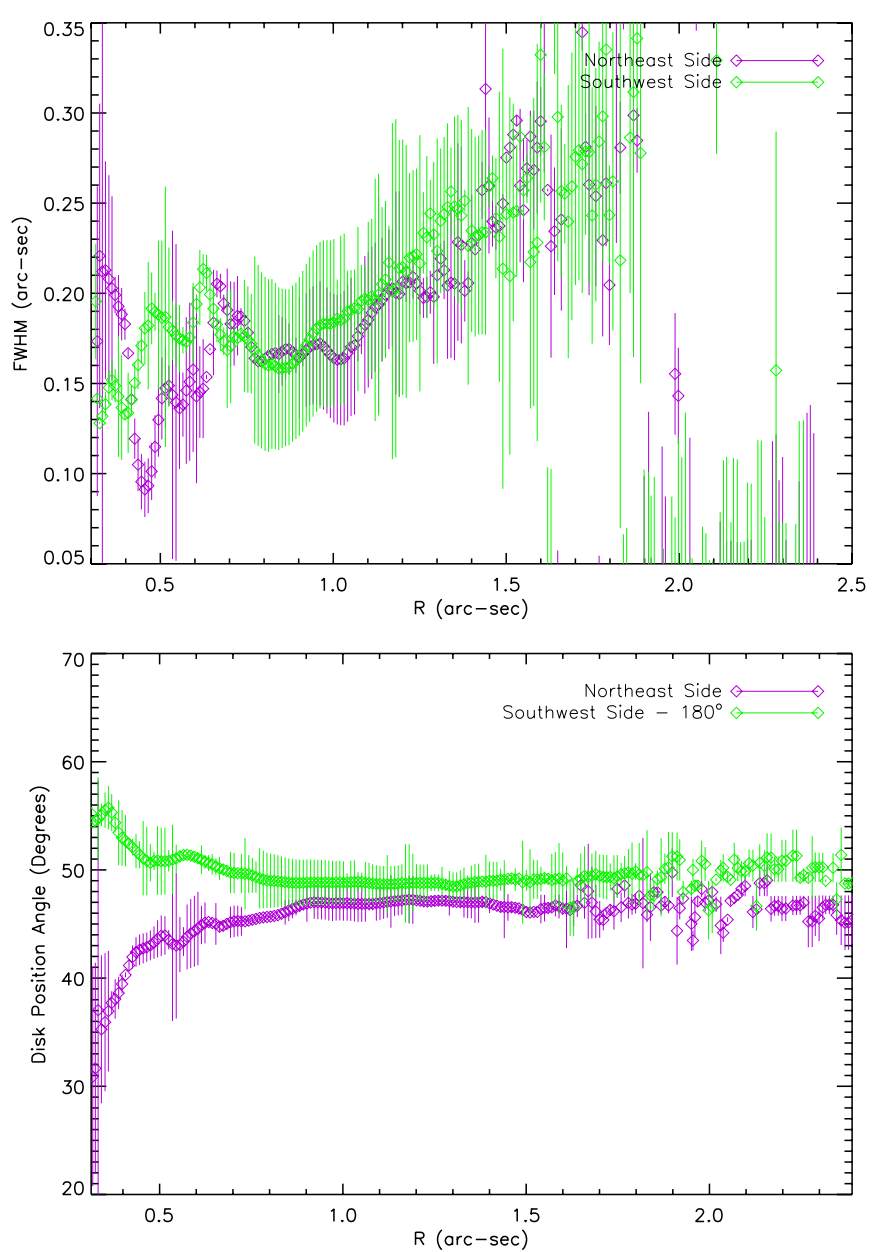

Figure 3. Disk FWHM (left) and position angle vs. angular separation for the NE and SW sides of the disk. The disk narrows at smaller angular separations. The two sides of the disk are offset in position angle by $\sim 3^{\circ}-4^{\circ}$; the disk curves toward the north starting at $r=0.9$.

(A color version of this figure is available in the online journal.)

\subsection{Disk Position Angle}

To quantify the "bow" structure easily seen in Figure 1 and identify any additional warping, we calculate the disk position angle as a function of stellocentric distance for both the NE and SW sides using the disk midplane pixel locations from the Gaussian fits described in Section 3.1. The position angle uncertainty at each radius results from the difference between the Gaussian-fitted disk midplane location at the radius and a midplane location defined by the brightest pixel. Here, we formally assume a systematic uncertainty of 0.009 from the Yelda et al. astrometric calibration, although fitting errors always dominate.

Beyond $r=0^{\prime} .9$, both sides of the disk maintain a constant position angle, although they are misaligned by 3-4 deg. Between 0'.3 and 0.'9, though, the disk emission on both sides curves toward the north, with the position angle decreasing on the NE side by more than $20^{\circ}$ and increasing by $\sim 10^{\circ}$ on the SW side. On the SW side, this curvature is not continuous, leveling off at $232^{\circ}$ at $0^{\prime \prime} .5-0^{\prime} .6$ before resuming at smaller separations. Beyond $r=11^{\prime \prime} 6$, the disk exhibits no obvious curvature/warping, though photon noise degrades the precision of our estimates. 


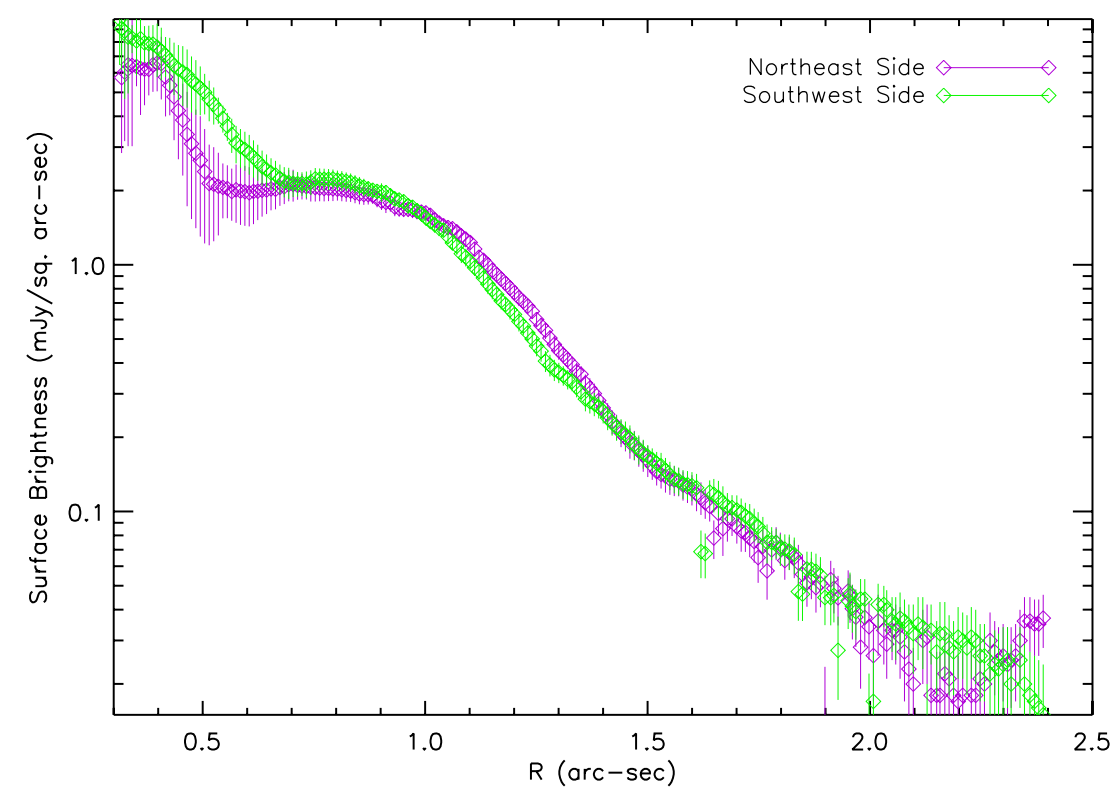

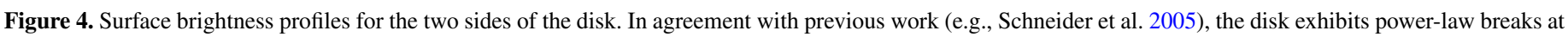
$r=1^{\prime \prime} .5$ and $r=1^{\prime \prime}$. 1 . We identify a strong jump in surface brightness starting at $r \approx 0$. .'5-0.'7.

(A color version of this figure is available in the online journal.)

\subsection{Disk Surface Brightness Profile}

To calculate the disk SB, we follow Rodigas et al. (2012) and determine the median SB in mJy $\operatorname{arcsec}^{-2}$ in an 18 pixel diameter circular aperture. We determine uncertainties in the median disk SB (also in mJy $\operatorname{arcsec}^{-2}$ ) at a given angular separation in a way analogous to that which we used to determine the disk $\mathrm{S} / \mathrm{N}$ per pixel. Specifically, at each pixel radius corresponding to a Gaussian-fitted disk midplane location, we calculate the uncertainty in SB within non-overlapping circular apertures identical in size to that which we use to determine the disk SB and covering all azimuthal angles exterior to the disk. We adopt the standard deviation of these SB estimates as the uncertainty in the disk SB at each radius.

Figure 4 displays the SB profiles for both sides of the disk. On both sides, the disk steadily brightens from $r=$ 2".5 to $r=1^{\prime \prime}$. However, at smaller separations, the profiles change dramatically. On the NE side, the disk has a roughly constant brightness at $0.5-0{ }^{\prime \prime} \cdot 9$ before brightening from 3 to $6 \mathrm{mJy} \operatorname{arcsec}^{-2}$ at 0.5 to $0^{\prime \prime} 4$. The SW side of the disk displays a similar behavior: a nearly constant brightness at $0.7-0.9$, a possible slight dip in brightness at $0{ }^{\prime \prime} .7$, and a sharp jump in brightness by a factor of four from $0{ }^{\prime \prime} .7$ to $0{ }^{\prime \prime} 4$. Thus, the disk SB profile appears "wavy" interior to $r=1$ ".

The two sides of the disk differ slightly in some other important respects. The locations of the peaks/plateaus closer to the star for the NE side than for the SW side. The shifted profiles are consistent with the dust ring being located at different stellocentric distances between the NE and SW sides: a pericenter offset. The NE side also plateaus and may drop slightly at $r \sim 0 ! 3-0 ! 35$. Conversely, on the SW side, the disk continues to brighten all the way to our inner working angle of $0 ! 3$.

Moreover, our analysis confirms evidence for a brightness asymmetry between the NE and SW sides. These differences are significant even at small separations where we detect the disk at a lower $\mathrm{S} / \mathrm{N}$. Interior to $0^{\prime} 6$, the median uncertainty in the disk SB is $\sim 2.8$ on the NE side and 4.6 on the SW side. While
Table 1

Surface Brightness Profile Power-law Indices

\begin{tabular}{|c|c|c|}
\hline $\begin{array}{l}\text { Angular Separation } \\
\left({ }^{\prime \prime}\right)\end{array}$ & $\begin{array}{c}a, b \\
(\mathrm{NE})\end{array}$ & $\begin{array}{c}a, b \\
(\mathrm{SW})\end{array}$ \\
\hline \multicolumn{3}{|c|}{ Single power law } \\
\hline$r=1-2.5$ & $2.13,-6.01$ & $1.72,-5.49$ \\
\hline \multicolumn{3}{|c|}{ Broken power law } \\
\hline$r=1.1-1.4$ & $2.23,-6.19$ & $\ldots$ \\
\hline$r=1-1.6$ & $\ldots$ & $1.78,-5.71$ \\
\hline$r=1.4-2.5$ & $1.31,-5.13$ & \\
\hline$r=1.65-2.5$ & $\ldots$ & $1.66,-5.33$ \\
\hline
\end{tabular}

Notes. Surface brightness profiles for the NE and SW sides determined by a Levenberg-Marquardt least-squares fit to the data. We assume a simple power law functional form of $f(X)=a \mathrm{X}^{b}$. Power laws in agreement with the data at the $95 \%$ confidence limit include values for $a$ that vary by $\sim 10 \%$ and values for $b$ that vary by $\sim 0.5$ dex.

these uncertainties are large, they are significantly smaller than the brightness differences at $r=0 .{ }^{\prime} 5-0{ }^{\prime} 6$, where the SW side is $2-3$ times brighter. At these angular separations, the $\pm 1 \sigma$ range in SB between the two sides do not overlap. The SW side of the disk is $50 \%$ brighter at $r \sim 0^{\prime \prime} 3-0^{\prime \prime} 4$, although the large error bars for the NE side SB make this difference less statistically significant.

We model the SB profiles over $r=0$ '! $3-2$ '. 5 to derive powerlaw indices $a$ and $b$ assuming a functional form of $f(X)=$ $a \mathrm{X}^{b}$. As a first guess, we take the logarithm of the SB, adopt uniform weighting to each data point, and estimate the power indices by fitting a straight line and deriving the slope. We then refine our power-law index estimates by performing a Levenberg-Marquardt minimization, while considering measurement errors. To more precisely estimate the locations of the power-law breaks, we perform the above steps iteratively, varying the break locations and adopting ones that minimize the reduced $\chi^{2}$ between the data and the model.

Table 1 summarizes our results. We fail to identify any power law to either lobe that quantitatively fits the entire radial extent 


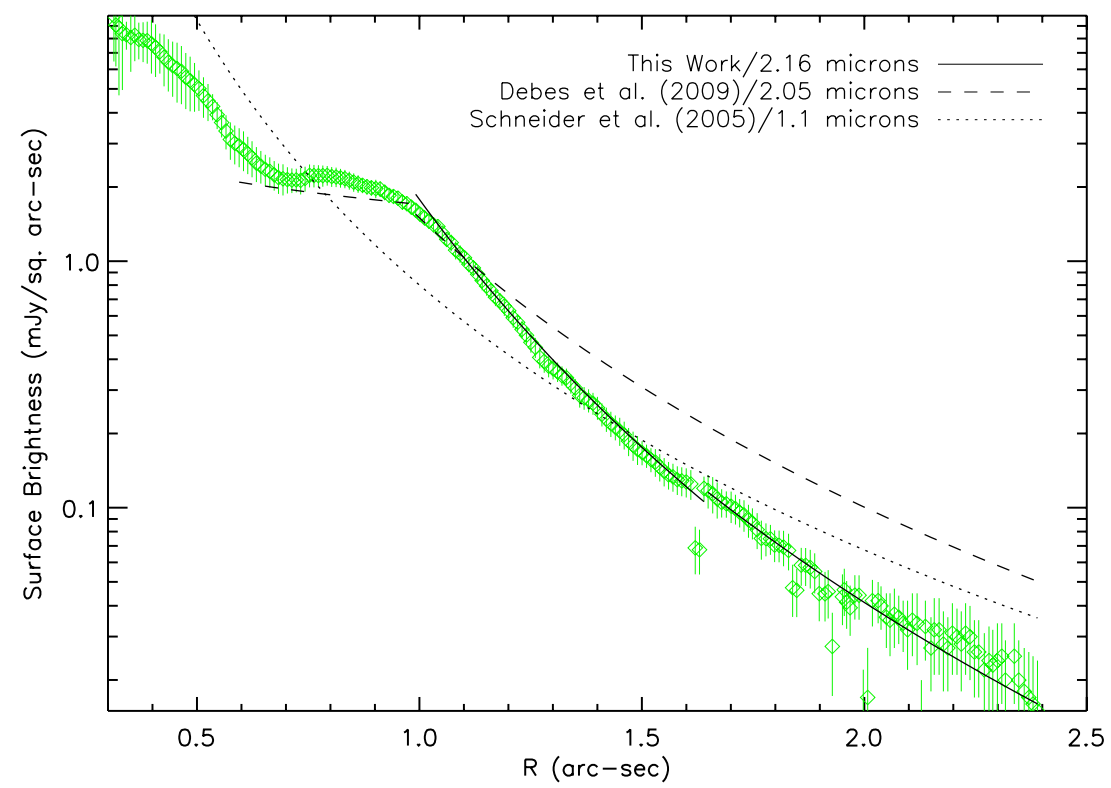

Figure 5. Comparisons between our best-fit power laws to the HD $32297 K_{s}$ surface brightness profile (SW side) and fitted power laws for 1.1-2.05 $\mu \mathrm{m}$ HD 32297 data from Schneider et al. (2005) and Debes et al. (2009). Our fits are generally much steeper. We find that the surface brightness profile interior to $r \sim 1^{\prime \prime}$ cannot be fit by a power law.

(A color version of this figure is available in the online journal.)

of the disk. For the NE (SW) side, the goodness-of-fit value $\left(R^{2}\right)$ declines to zero at $r_{\text {in }} \sim 1^{\prime \prime} .0(0$.'9). Assuming a single power law for both sides, we derive best-fit coefficients of $a=2.13, b=$ -6.01 at $r=1^{\prime \prime} .05-2.5$ for the NE side and $a=1.72, b=-5.49$ for the SW side at $r=0$ '.95-2'.5. However, the goodness-of-fit criterion for the NE side is low $\left(R^{2} \sim 0.75\right)$, indicating that at least this side may be best modeled as a broken power law (see also Schneider et al. 2005; Debes et al. 2009).

For the NE side, the best-fit indices assuming a broken power law are $a=2.23, b=-6.19$ at $r=1^{\prime \prime} .1-1^{\prime \prime} .4$ and $a=1.31$, $b=-5.13$ at $r=1^{\prime \prime} .4-2$ ".5. For the SW side, the best-fit indices are similar: $a=1.78, b=-5.71$ at $r=1^{\prime \prime}-1^{\prime \prime} .6$ and $a=1.66$, $b=-5.33$ at $r=1.65-2.5{ }^{11}$ Formally, our values for $b$ have a large uncertainty since the goodness-of-fit criterion remains above 0.95 for $a \pm 20 \%$ and $b \pm 0.5$.

We can compare our SB profiles to those from Schneider et al. (2005) and Debes et al. (2009) to understand how the profiles change with wavelength. In general, the shape of the SB profiles show good agreement with those derived from $1.1 \mu \mathrm{m}$ data by Schneider et al. (2005). Although they do not draw attention to any steep increase in SB at $r=0^{\prime \prime} .5-0^{\prime \prime} .7$, their Figure 2 provides some evidence for this feature, at least on the SW side. While we do not find evidence for a steep drop in SB at $r=0$ 0.3-0.'5 for the NE side as they do, the SB does plateau at $r=0.4$ and drop slightly. The $2.05 \mu \mathrm{m}$ profile from Debes et al. (2009) does not extend to $r<0$ '.5-0.' 6 , so we cannot know whether they too find evidence for a jump in SB at small separations. Our profiles are significantly steeper than those from Schneider et al. (2005) and Debes et al. (2009) at $r>1^{\prime \prime}$ obtained at shorter wavelengths (Figure 5).

\footnotetext{
11 While the inner and outer fitted radii reported here are the ones for which a power-law fit is most applicable, we obtain similar results for slightly different radii: e.g., for $r_{\text {in }}=0.95$ and $r_{\text {out }}=1^{\prime \prime} .65$.
}

\section{DEBRIS DISK MODELING}

\subsection{Scattered Light Modeling}

To understand the disk's grain properties and morphology, we compare our image to synthetic resolved disk models with a range of scattering properties. We modeled a number density distribution of dust for the disk in the following cylindrical form, which allows for a variety of disk morphologies:

$$
\begin{aligned}
N(r, z) & =\exp \left(\frac{r-r_{\mathrm{o}}}{2 \sigma_{r}^{2}}\right) \exp \left(\frac{z}{2 \sigma_{z}^{2}}\right), r<r_{\text {break }} \\
& =\left(\frac{r}{r_{\text {break }}}\right)^{-\beta}, r \geqslant r_{\text {break }},
\end{aligned}
$$

where $r_{\mathrm{o}}$ can be interpreted as the location of a birth ring of planetesimals generating a collisional cascade of dust with a characteristic width $\sigma_{r}$. We assume a Gaussian scale height to the disk at all radii, and we allow for a power-law decrease in dust density beyond some radius $r_{\text {break }}$, thus allowing for combinations of ring-like and disk-like structures. We place dust particles at various distances from the star following the density distribution above and distribute them uniformly in the azimuthal angle $(\theta)$. To derive the integrated SB, we project the density distribution on a two-dimensional plane after transforming the coordinates of dust particles to account for inclination and position angle and scale the result to the observed $\mathrm{SB}$ of the disk in the Keck images. The SB, $F$, of each particle is determined from its distance from the star and its scattering angle $\omega$, assuming a two component Henyey-Greenstein phase function:

$$
\begin{aligned}
F & \propto\left(\frac{1}{r^{2}+z^{2}}\right)\left[a_{1} \frac{1-g_{1}^{2}}{\left(1+g_{1}^{2}-2 g_{1} \cos \omega\right)^{1.5}}\right. \\
& \left.+a_{2} \frac{1-g_{2}^{2}}{\left(1+g_{2}^{2}-2 g_{2} \cos \omega\right)^{1.5}}\right] .
\end{aligned}
$$




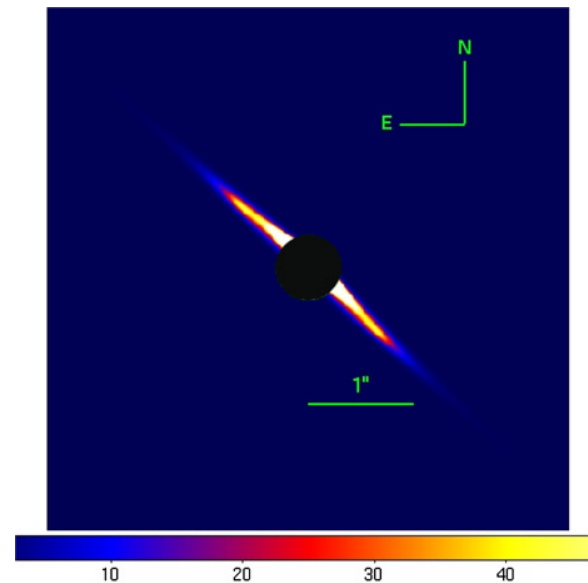

10 20 30 40

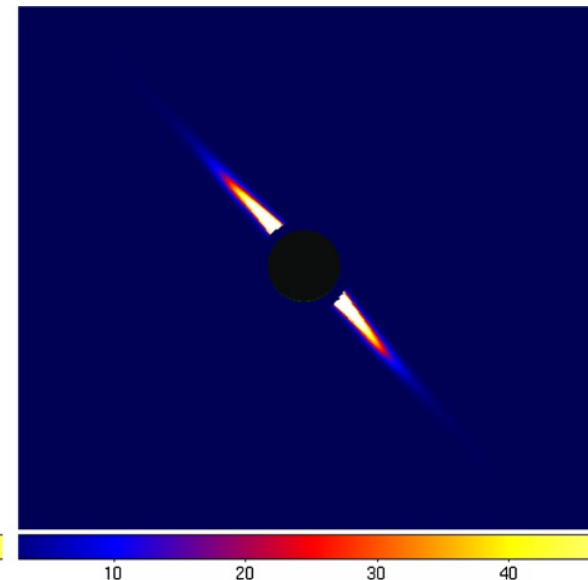

10

20

30

40
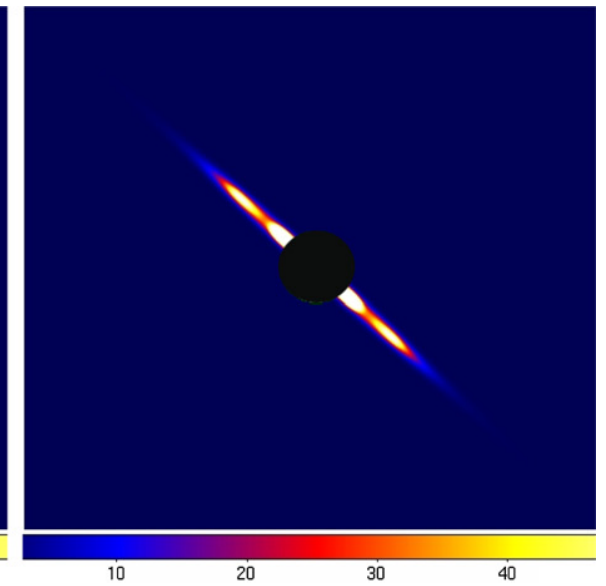

6. Scattered light models incorporating different grain properties/disk geometries: (left) a two-component Henyey-Greenstein model with strongly forward

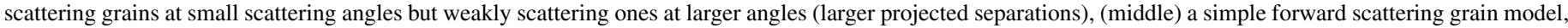
(right) two isotropically scattering dust rings. The left-hand model best reproduces the disk SB profiles. The units are in counts.

(A color version of this figure is available in the online journal.)

Table 2

HD 32297 Scattered Light Model Parameters

\begin{tabular}{lc}
\hline \hline Parameter & Value \\
\hline$r_{\mathrm{O}}$ & $110 \mathrm{AU}$ \\
$\sigma_{\mathrm{r}}$ & $13 \mathrm{AU}$ \\
$\mathrm{FWHM}_{\mathrm{r}}$ & $30 \mathrm{AU}$ \\
$\sigma_{\mathrm{z}}$ & $2 \mathrm{AU}$ \\
$\mathrm{FWHM}_{\mathrm{z}}$ & $5 \mathrm{AU}$ \\
$r_{\text {break }}$ & $110 \mathrm{AU}$ \\
$g_{1}$ & 0.96 \\
$a_{1}$ & 0.9 \\
$g_{2}$ & -0.1 \\
$a_{2}$ & 0.1 \\
$\beta$ & 3.4 \\
$i$ & $20^{\circ}$ \\
Position angle & 47.5 \\
\hline
\end{tabular}

A multi-component phase function for circumstellar dust may be favored. For example, the phase function of zodiacal dust in the solar system has been modeled with multiple Henyey-Greenstein components, including a significant backscattering component (Hong 1985). Recently, observations of HR 4796A's disk SB as a function of scattering angle showed an incredibly flat inferred phase function for its dust at scattering angles $>50^{\circ}$ (Dalle Ore et al. 2011), and observations of the protoplanetary disk HD 100546 at multiple wavelengths require forward scattering grains where the phase function becomes flatter at scattering angles $>38^{\circ}$ (Mulders et al. 2012).

Figure 6 displays the model that best reproduces the overall disk morphology and two rejected models. We attempted several possible structures and combinations of parameters, obtaining a good fit to the data with the model presented in Table 2 and shown in the left panel. A more rigorous exploration of parameter space and their possible degeneracies is beyond the scope of this paper.

To account for its warped, bow-shaped appearance, the disk must contain some highly forward scattering grains at $r \sim$ $110 \mathrm{AU}$, which cause a brightness asymmetry between the front and back sides of the disk. At low scattering angles (small projected separations), the brightness asymmetry is more pronounced than at larger projected separations, causing a change in the midplane position angle. This type of "warping" structure is also seen in HD 15115 (Debes et al. 2008; Rodigas et al. 2012). However, the sharp breaks in the SB at $\sim 1^{\prime \prime}$ are hard to reproduce with typical single-component Henyey-Greenstein phase functions (middle panel), requiring a flatter phase function at larger scattering angles (left panel). The SB breaks could conceivably be reproduced by a superposition of two rings of isotropically scattering dust (right panel), but such a configuration does not give rise to the disk's warped appearance. We cannot exclude the presence of a second inner ring (i.e., at $r \sim$ 35-50 AU) for our modeled dust phase function, especially if the dust in the disk at wider separations is slightly less forward scattering than we have modeled. This would allow the inner disk to dominate the SB at small projected separations.

Although our two-component forward scattering model is simple, it reproduces some key disk features. Figures 7 and 8 (left panel) compare this model to the observed SB profile. The model clearly succeeds in reproducing the disk warping at $r<1^{\prime \prime}$ and the SB profile break/turnover at $\sim 110$ AU. The model grains' strong forward scattering at small angles causes the disk to appear very bright again at small projected separations $\left(r<00^{\prime} 6\right)$. This feature agrees with our measured SB profile (Figure 8), though taken at face value the model predicts that this brightness accelerates, whereas the measured profile appears to flatten at $r \sim 00^{\prime} 4$, especially for the NE side. Furthermore, our scattered light model predicts that the disk emission should be roughly axisymmetric. However, at $r<0$ ' 6 , the disk's SW side is significantly brighter and the profile breaks appear offset.

The disk's asymmetric SB profile breaks could indicate an asymmetry in the disk's distance from the star as a function of position angle: e.g., a pericenter offset. To investigate whether a pericenter offset better reproduces the SB profile, we reconstruct a scattered light model with identical grain scattering properties as our two-component model but make the SW side of the disk 5-10 AU closer to the star than the NE side (Figure 8). This model predicts that the NE side's SB profile break starts at wider separations ( $r \sim 1^{\prime \prime}$. 1 versus $0{ }^{\prime} 9$ for the SW side) and that the SW side should be brighter at 0.'5-0!'9, in agreement with the observed SB profiles.

The pericenter offset model's fidelity is not perfect: the SW side is significantly underluminous and the exact locations of the breaks are not well reproduced. However, its success in 


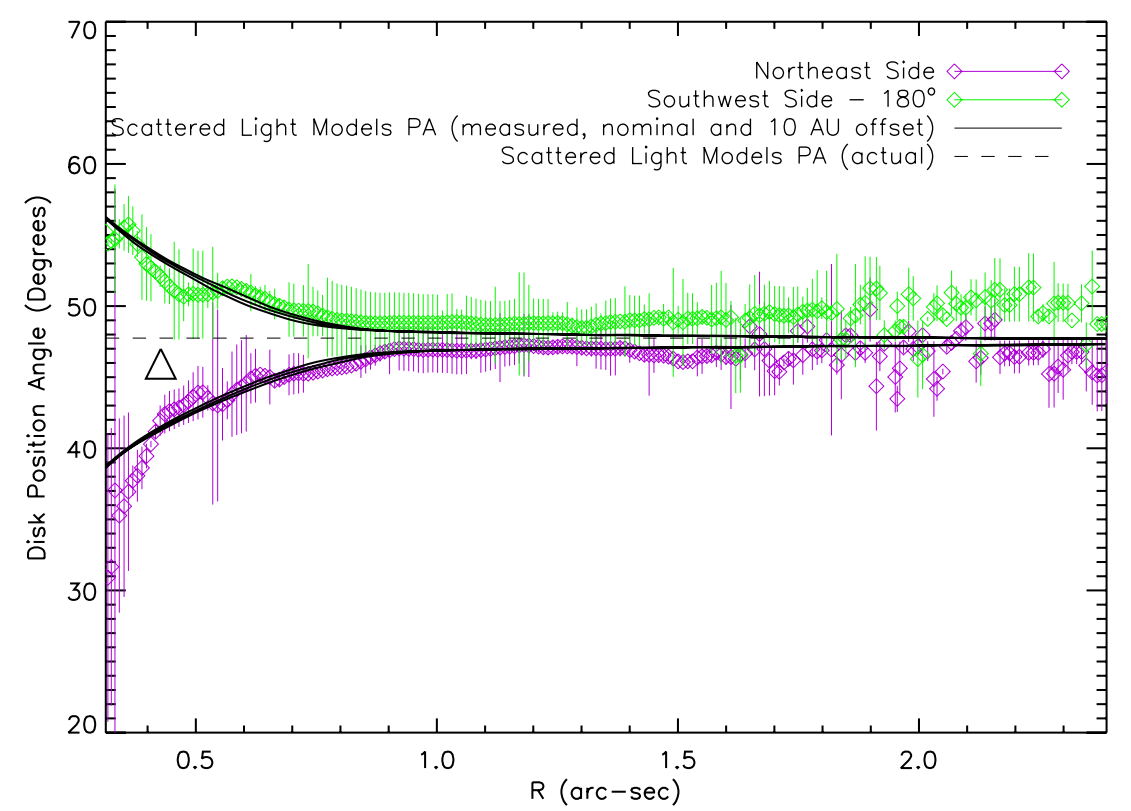

Figure 7. Comparisons between the modeled and measured disk position angles (right). The triangle identifies the location of the mm brightness peak from Maness et al. (2008). We use the two-component model with the dust ring centered on the star and with a $10 \mathrm{AU}$ offset.

(A color version of this figure is available in the online journal.)
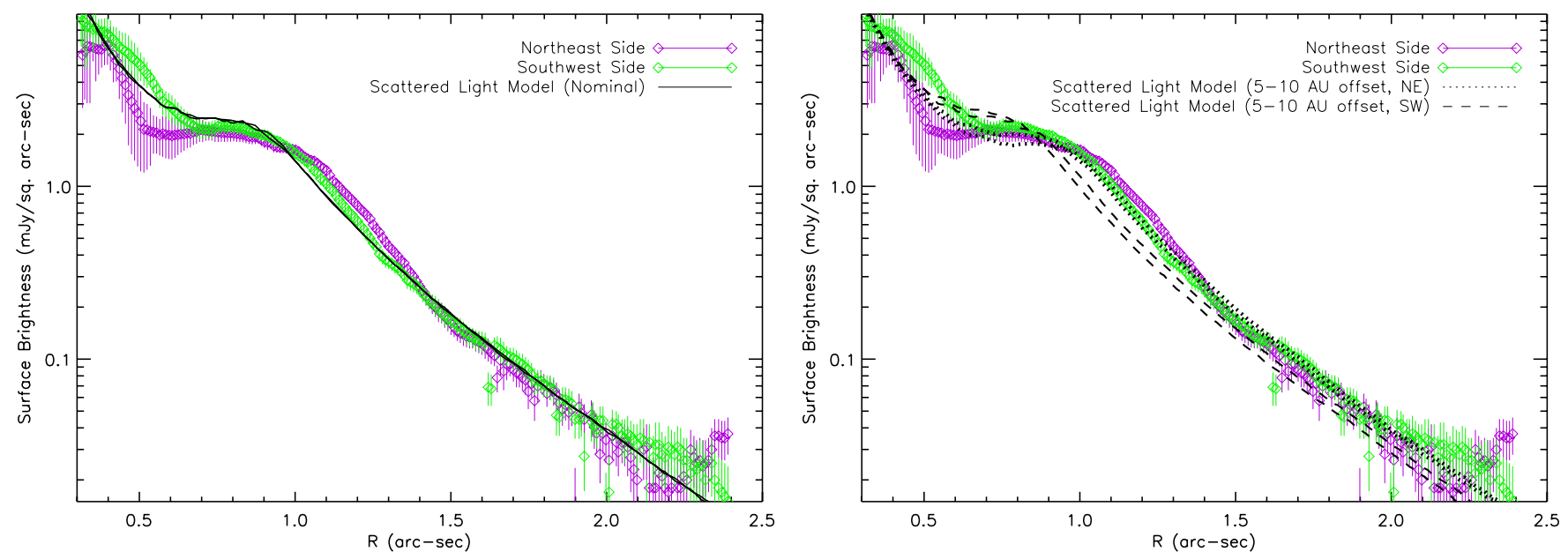

Figure 8. Comparisons between the modeled and measured surface brightness profiles for a dust ring centered on the star (left) and one with a 5-10 AU pericenter offset (right; the SW side is 5-10 AU closer). Both models reproduce the wavy SB profile. The offset makes the disk model qualitatively reproduce the differences in the observed SB peaks/plateaus at $r<1^{\prime \prime}$, although it degrades the model's fidelity at $r>1^{\prime \prime}$, especially on the SW side, where it is substantially underluminous.

(A color version of this figure is available in the online journal.)

reproducing the asymmetric SB profile breaks motivates more detailed scattered light disk modeling. In particular, all single ring models (regardless of any pericenter offset) predict that the disk's sides should be equally luminous at $r \sim 0$ '!3-0'.4. Our data indicate otherwise (the SW side is still brighter by $\sim 50 \%$ ), suggesting the need for further modifications for our scattered light model. New, higher S/N images of HD 32297's disk will clarify the angular separation range over which the SW side is brighter and thus provide important constraints for future disk modeling.

\subsection{Spectral Energy Distribution Modeling}

For a separate but related probe of the HD 32297 disk properties, we modeled the disk spectral energy distribution
(SED) from point-source photometry including newly available data from the Spitzer Space Telescope, WISE mission, and AKARI satellite (Werner et al. 2004; Wright et al. 2010; Murakami et al. 2007). Table 3 lists the photometric data we consider.

Our methods follow those outlined in Plavchan et al. (2009), where we identify the best-fit dust temperature(s), grain properties, and effective emitting areas incorporating a downhill simplex algorithm ("Amoeba") as described in Press et al. (1992). We consider cases where the dust radiates like a blackbody such that $r_{\text {dust }, \mathrm{AU}}=(280 \mathrm{~K} / \mathrm{T})^{2} \times\left(L_{\mathrm{star}} / L_{\odot}\right)^{1 / 2}$ and where the dust's emissivity scales with the effective grain size beyond a critical wavelength (Backman et al. 1992), $\epsilon \propto \lambda^{-\beta}$, which can place grains of a given temperature at larger distances. We solve 
Table 3

HD 32297 Photometric Data

\begin{tabular}{lccl}
\hline \hline Filter/Wavelength & $\begin{array}{c}\text { Flux } \\
(\mathrm{mJy})\end{array}$ & $\begin{array}{c}\sigma \text { Flux } \\
(\mathrm{mJy})\end{array}$ & \\
\hline$B / 0.4380$ & 1836.74 & 28.75 & TYCHO-II(trans) \\
$V / 0.545$ & 2012.91 & 24.10 & TYCHO-II(trans) \\
$J / 1.235$ & 1341.80 & 34.96 & 2MASS \\
$H / 1.662$ & 913.48 & 50.58 & 2MASS \\
$K_{s} 2.159$ & 611.41 & 11.95 & 2MASS \\
3.37 & 280.23 & 8.21 & WISE \\
4.62 & 151.28 & 3.78 & WISE \\
8 & 65.18 & 2.75 & IRSep \\
11.2 & 49.9 & 2.1 & Fitzgerald et al. (2007) \\
11.66 & 53 & 5.3 & Moerchen et al. (2007) \\
12.08 & 55.25 & 1.20 & WISE \\
16 & 71.28 & 2.53 & IRSep \\
18.3 & 90 & 13.5 & Moerchen et al. (2007) \\
22.19 & 212.99 & 5.55 & WISE \\
24 & 225.2 & 4.82 & IRSep \\
70 & 850 & 60 & IRSep \\
90 & 823.2 & 116 & AKARI \\
160 & $<460$ & & IRSep \\
1300 & 5.1 & 1.1 & CARMA/Maness et al. (2008) \\
\hline
\end{tabular}

Notes. TYCHO-II(trans) refers to TYCHO-II catalog data transformed into the standard Johnson-Cousins photometric system. IRSep refers to the IRSenhanced product data set as queried from the NASA/IPAC Infrared Science Archive.

for the disk model parameters that minimize the fit residuals relative to the flux $\left(\mathrm{rms}_{\mathrm{rel}}=\left(\left(\sum_{i=0}^{n} \Delta_{i} / \mathrm{Flux}_{i}\right)^{2} / N\right)^{1 / 2}\right)$. In all cases, we assume that the grain populations have a characteristic size; for simplicity, we do not consider a grain size distribution.

Even though we include photometry not previously modeled, our fits are likely to be highly degenerate (e.g., Maness et al. 2008; Fitzgerald et al. 2007). Therefore, instead of identifying the single best-fit model and $95 \%$ confidence interval, we use several separate SED model runs to explore more focused questions about the HD 32297 disk properties.

1. Model 1: a single dust population. Here, we assume that only one dust population contributes to the disk's IR-to-mm emission and allow $\beta$ to vary. Our goal with this fit is to determine whether HD 32297's disk must have more than one dust population.

2. Model 2: two dust populations, one dust belt at a fixed location. Here, we include two dust populations and allow their emissivity laws to vary but fix them to the same location. We place the belt at $r=85 \mathrm{AU}$, or roughly interior to where the disk SB profile begins to flatten. Our goal here is to determine whether more than one dust location is required.

3. Models 3-5: two dust populations/belts, fixed emissivity. Here, we identify the best-fitting model with two dust populations in two distinct belts, assuming either that the grains behave as blackbodies or have a $\lambda^{-1}$ emissivity law. This approach follows that of Maness et al. (2008) and will allow us to assess whether their formalism provides a better match to the photometry than assuming one dust population/belt.

4. Model 6: two dust populations/belts, variable emissivity, fixed location. Here, we fix the locations of the dust to the peak of the SB profiles: $\sim 0$.' 4 and $\sim 1^{\prime \prime}$ or $45 \mathrm{AU}$ and $110 \mathrm{AU}$, which roughly cover the locations of the $\mathrm{SB}$ plateaus in the NE and SW sides. Our goal here is to see whether we can identify a good-fitting disk model that identifies the local maxima in the SB profile as the locations of two separate debris belts.

5. Models 7-9: three dust populations/belts, variable emissivity, variable/fixed location. Maness et al. (2008) suggest that three separate dust populations are needed to fit the HD 32297 SED. In one case, we fix all the belts to be at $85 \mathrm{AU}$ and incorporate a third, warmer dust population varying in grain size and emissivity. In two cases, we fix the outer two dust belts at $45 \mathrm{AU}$ and $110 \mathrm{AU}$, while varying grain size and emissivity, and incorporate a third warmer dust population varying in radius, grain size, and emissivity.

Figure 9 displays some of our SED modeling results, which are reported in Table 4. For all best-fit models, the stellar effective temperature is $T_{\star} \sim 7870-7890 \mathrm{~K}$ and the star has little extinction $\left(A_{V}<0.03\right)$. Regardless of our assumed particle emissivity law, a single dust population model (Model 1, top-left panel) poorly reproduces the SED, as was found previously (Fitzgerald et al. 2007), significantly underpredicting the flux density at $8-22 \mu \mathrm{m}$. Formally, having two or three dust populations in one belt at $85 \mathrm{AU}$ (i.e., top-right panel) significantly improves the fit $\left(\mathrm{rms}_{\text {rel }}=0.05-0.08\right)$. However, the required grain sizes are too small $(\sim 4 \mathrm{~nm}-0.2 \mu \mathrm{m})$ to be realistic. Thus, the dust likely arises from more than one singletemperature dust population with given grain size and emissivity power law.

Table 4

HD 32297 SED Modeling Results

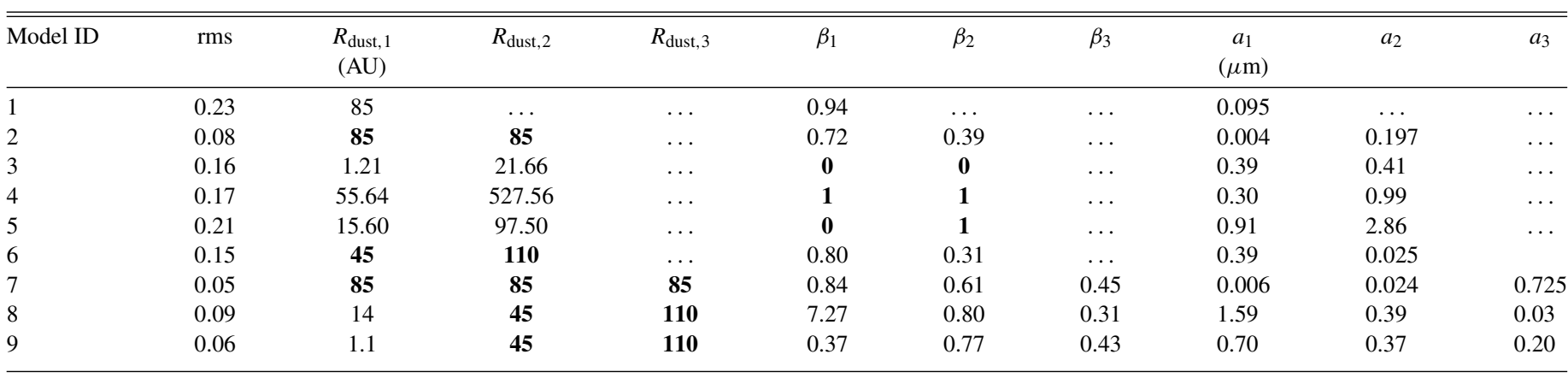

Notes. rms refers to the fit residuals relative to the flux $\mathrm{rms}_{\text {rel }}=\left(\left(\sum_{i=0}^{n} \Delta_{i} / \mathrm{Flux}_{i}\right)^{2} / N\right)^{1 / 2}$, where $N$ is the number of flux density measurements $(18) . R_{\mathrm{dust}}$ refers to the dust ring's stellocentric distance, $\beta$ is the particle emissivity power law, and $a$ is the grain size in microns, where $2 \pi a=\lambda_{o}$ (see Backman et al. 1992). Values in bold are fixed for a given model run, whereas others are "fitted" values. 

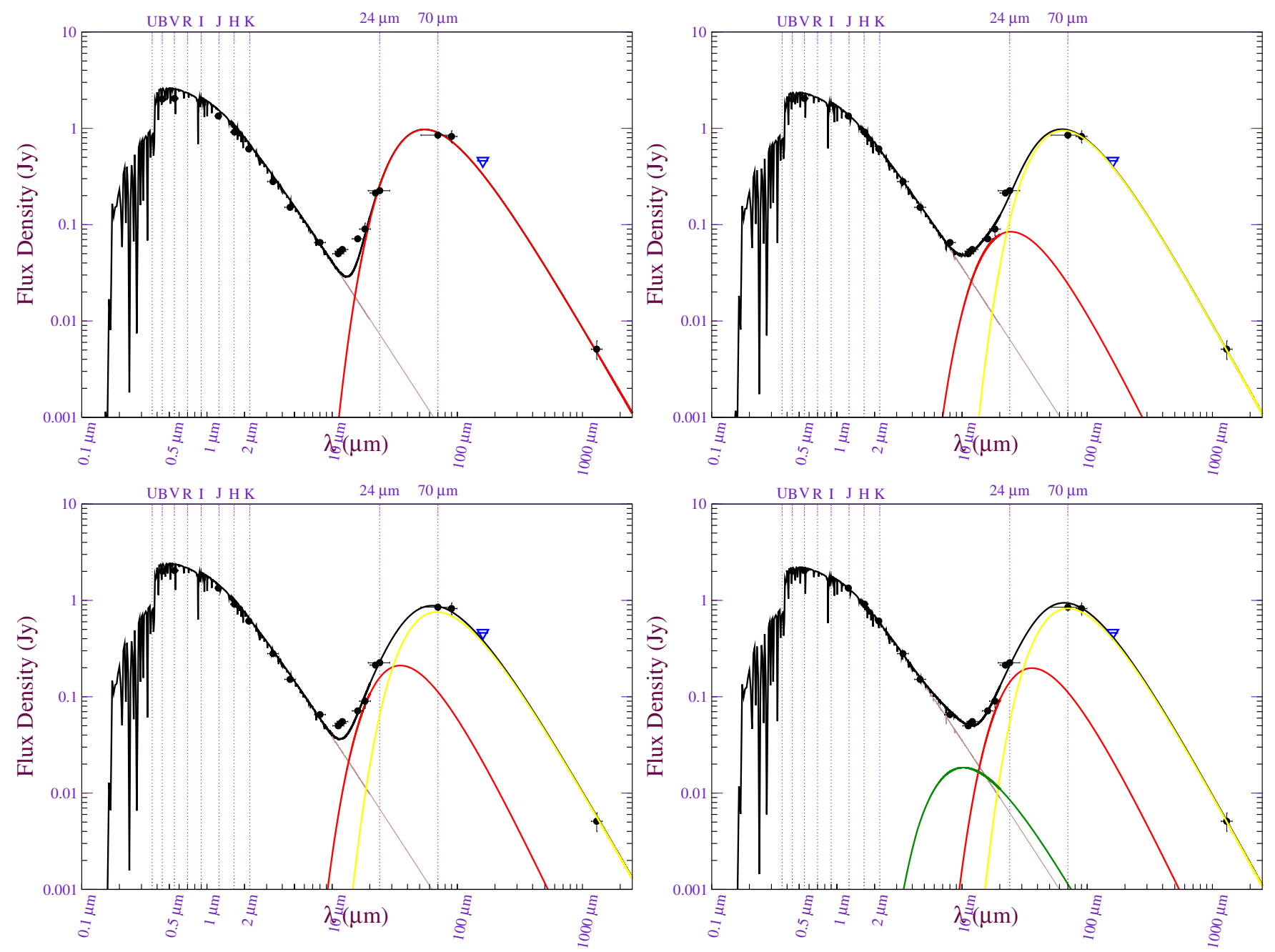

Figure 9. SED model fits to the HD 32297 photometric data listed in Table 3: Model 1 (top left), Model 2 (top right), Model 6 (bottom left), and Model 9 (bottom right).

(A color version of this figure is available in the online journal.)

Fits assuming blackbody grains but incorporating two dust components at different locations significantly improve the fit $\left(\mathrm{rms}_{\text {rel }} \sim 0.16\right.$; top right $)$ compared to a single dust component model. The fits imply that the dust responsible for $10-1000 \mu \mathrm{m}$ emission is at 1.2-22 AU or $r \sim 0^{\prime}$ '01-0'.2, but Fitzgerald et al. (2007), Moerchen et al. (2007), and Maness et al. (2008) show that the disk emission instead originates on scales more comparable to $\approx 30-100 \mathrm{AU}$. The broad range of orbital radii is indicative of the significant model degeneracies involved. Assuming that at least one of the dust components has an emissivity of $\beta=1$ yields fits with larger grains $(0.3-3 \mu \mathrm{m})$ but worsens the fit $\left(\mathrm{rms}_{\mathrm{rel}} \sim 0.17-0.21\right)$. Thus, if the dust disk geometries approximate thin, isothermal rings and consist of grains dominated by a single, characteristic size, then the grains likely have emissivity power laws somewhere between zero and one.

The bottom panels of Figure 9 show that it might be possible for the debris rings to reside at locations equal to the scattered light brightness peaks $(r \approx 45 \mathrm{AU}, 110 \mathrm{AU})$ and reproduce the IR-to-mm disk emission. A two dust population model (Model 6; bottom left) reproduces the SED well at 1-8 $\mu \mathrm{m}$ and $17-1300 \mu \mathrm{m}$ but underpredicts the disk emission at $\sim 12 \mu \mathrm{m}$ by $\sim 50 \%$. Starting with the Model 6 results and incorporating a third dust population, we reproduce the SED if the dust is at $\sim 14 \mathrm{AU}(171 \mathrm{~K})$, although the emissivity power law for this population is unphysical $(\beta \sim 7.3)^{12}$ and the grain sizes for the coldest dust component are too small (Model 8). However, allowing the emissivity law and grain sizes for all three components to vary yields an excellent fit to the data (rms $\sim 0.06)$. The emissivity laws and grain sizes for this model (Model 9; bottom right) are reasonable ( $\beta=0.37-0.77$; $a=0.20-0.70 \mu \mathrm{m})$. Here, the third component is at $\sim 1 \mathrm{AU}$ with a temperature of $\approx 430 \mathrm{~K}$ and is responsible for the weak $8 \mu \mathrm{m}$ excess and substantially contributes to the disk emission at $12-17 \mu \mathrm{m}$. Thus, although it is not necessary, it is at least possible to identify the SB profile peaks at $45 \mathrm{AU}$ and $110 \mathrm{AU}$ with separate thin dust rings responsible for broadband emission at 8-1300 $\mu \mathrm{m}$, provided that there exists an unresolved, warmer dust component located interior to $45 \mathrm{AU}$.

We emphasize that the model fits we report in Table 4 are only some of many possible fits, the range of which is further limited by our input assumptions about the disk. For example, because our model is set up only to consider infinitely thin, isothermal rings, it is unclear whether the warmer dust emission

\footnotetext{
12 This model run finds $\beta \sim 7.3$ as the "best-fit" model because the Amoeba code treats $\beta$ as a true free parameter, regardless of whether the value is physical, and exploits a gap in SED coverage at $\sim 10 \mu \mathrm{m}$ to achieve a better fit.
} 
we identify originates in a separate ring or rather an annulus, the outer edge of which we see at wider separations. Given the extreme number of model degeneracies, the most we can say is that (1) the debris emission must originate from more than one population at (2) multiple locations and (3) among the many possible configurations, the dust populations may coincide with the SB peaks. To make further progress, we need high spatial resolution, high $\mathrm{S} / \mathrm{N}$ imaging of the disk at a wider range of wavelengths to derive much more stringent constraints on the dust sizes/scattering properties and location(s) of the dust population(s) (e.g., Debes et al. 2009; Rodigas et al. 2012).

\section{COMPARISONS TO PREVIOUS HD 32297 RESULTS}

From optical coronagraphic imaging, Kalas et al. (2005) find evidence for warping in the HD 32297's disk, especially for the NE side, where the disk at $r>500-600$ AU appears swept back compared to midplane regions at smaller angular separations. They attribute the warp to ISM sculpting of the disk as it moves south through ISM material. The warped structure we resolve curves in the opposite direction from that expected due to ISM sculpting (see images for HD 32297/15115/61005 from Debes et al. 2009) and does so for both sides of the disk. Furthermore, ISM sculpting should be prominent only at larger separations in our images, where the disk is weakly detected $(r>1$ '.5), because the grains need time to become entrained in the ISM flow (e.g., Debes et al. 2009). However, optical and near-IR imaging probe two very different characteristic grain populations, and the size scales over which we resolve the disk do not overlap. Thus, our results are not in conflict. Rather, ISM sculpting may explain the optical image but does not explain the near-IR image.

We confirm and clarify three major claims from previous nearIR imaging (Schneider et al. 2005; Mawet et al. 2009; Debes et al. 2009). First, we verify the brightness asymmetry found by Schneider et al. (2005) and Mawet et al. (2009) at small $(r \sim 45-55 \mathrm{AU})$ separations and show that it persists down to $r \sim 35 \mathrm{AU}$, albeit at a lower statistical significance. Second, we recover a break/deviation in the SB power law at $r \sim 100 \mathrm{AU}$ found by Debes et al. (2009). Our new results show that the SB profile at smaller separations does not follow an extension of the power law describing the disk at larger separations: instead, it appears wavy. Finally, Mawet et al. (2009) claim that the NE side of the disk emission is truncated at $r \sim 0$.' 6 . We find that the NE SB profile flattens (not brightens) from $r \sim 1^{\prime \prime}$ to $r \sim 0$ '.5. Assuming that their coronagraph attenuates some flux at separations slighter greater than their inner working angle $(0.4)$, most of the NE side emission will be hidden from their view. In contrast, the SW side clearly begins to brighten at wider separations ( $r \sim 00^{\prime} .5-0^{\prime \prime}$. $)$ visible by Mawet et al. (2009). Thus, our results agree.

We also note that a separate work in preparation confirms these results. In particular, S. Esposito et al. (2012, in preparation) analyze the HD 32297 disk separation Keck/NIRC2 obtained in $\mathrm{H}$ and $\mathrm{K}_{\mathrm{s}}$. They identify the warping/bowing of the disk in both data sets, confirming our discovery and demonstrating that this disk feature is present at multiple wavelengths.

Thermal IR imaging from Fitzgerald et al. (2007) and Moerchen et al. (2007) shows that the HD 32297 disk has an inner clearing devoid of grains slightly larger than those we can probe with our data. Fitzgerald et al. find that the disk exhibits a bilobed structure and most of its $11.2 \mu \mathrm{m}$ emission originates from $r \sim 0$.'5-0.' 6 , characteristic of a dust ring with an inner edge at $\approx 60 \mathrm{AU}$. Moerchen et al. resolve the disk at $12 \mu \mathrm{m}$ and $18 \mu \mathrm{m}$ out to slightly wider separations $\left(r<1^{\prime \prime} .3\right)$.
Based on the disk's brightness temperature, they likewise find evidence for an inner clearing, albeit one that is slightly larger $(r \sim 80 \mathrm{AU})$. The plateaus in our SB profiles could identify the inner boundaries of dust populations (e.g., Rodigas et al. 2012). Under this interpretation, our image is consistent with a dust belt truncated at $r \sim 1^{\prime \prime}(\approx 110 \mathrm{AU})$ and a second one at $\approx 0$. 4-0.'6 (45-70 AU). Because these authors did not analyze their data in the same way, it is difficult to compare their results between each other and their combined results against ours. Still, their results and ours are qualitatively consistent with the HD 32297 disk having at least one dust population truncated at small separations.

In agreement with Maness et al.'s (2008) mm study, we find evidence for at least two dust grain populations, responsible for the mid-IR excess emission and far-IR/submillimeter excess, respectively (see also Moerchen et al. 2007). They are able to fit the SED from 1 to $100 \mu \mathrm{m}$ but not $1300 \mu \mathrm{m}$, a discrepancy they explain by adding a third, cold dust population. Our modeling generally fits the SED from $17 \mu \mathrm{m}$ to $1300 \mu \mathrm{m}$ but slightly underpredicts emission at $12 \mu \mathrm{m}$, a discrepancy we can solve if there exists an additional, unresolved warm dust population. Our different results are likely a byproduct of our modeling assumptions: they allow the dust populations to be spatially extended but fix the particle emissivity law, whereas we assume the dust is confined in isothermal rings but allow the emissivity law to vary.

As noted in Section 3, the peak of HD 32297's mm emission from Maness et al. lies close to the bright inner disk region on the SW side. While formally the position angle $\left(\approx 46^{\circ}\right)$ of the $\mathrm{mm}$ emission is offset by $\approx 10 \mathrm{deg}$, it tracks the disk's position angle at wider separations much better. The disk's warped appearance at $r<1^{\prime \prime}$ is due to its strong forward scattering at small angles. If the disk's grains instead isotropically scattered starlight, its $K_{s}$ emission would lie almost directly on top of the mm peak. Thus, we identify the $\mathrm{mm}$ and near-IR brightness asymmetry as originating from the same location. Assuming the grains responsible for both the $\mathrm{mm}$ and near-IR emission are likely the result of collisions, they may trace the same parent population of planetesimals.

\subsection{Summary and Future Work}

Using Keck/NIRC2 $K_{s}$ coronagraphic imaging, we resolve the HD 32297 disk at a high $\mathrm{S} / \mathrm{N}$ from $r=0$ '!3 to $r=2$ ". 5 . We determine basic disk properties (SB, FWHM, position angle), compare our image to disk models with a range of (grain) scattering properties, and model newly available, broadband photometry to provide a complementary investigation of HD 32297's circumstellar environment. We obtain the following major results.

1. We discover that HD 32297's debris disk exhibits a prominent warped or "bow"-shaped structure interior to $r \sim 1^{\prime}$ ( $110 \mathrm{AU})$.

2. Our new data clarify the disk's SB profile at small separations. We find that it has a "wavy" profile interior to $r \sim 110 \mathrm{AU}$, with a plateau extending to $r \sim 0.5-0.7$ (55-80 AU) before the disk brightens by factors of 3-4 at smaller separations.

3. The disk exhibits significant asymmetries between the two sides. The SW side is brighter at $r \sim 0$.'3-0.' 6 by $50 \%-150 \%$, with the most statistical differences being at $r \sim 0$.5-0.'6. These separations are roughly where the disk's mm emission peaks and are consistent with previous 
results (e.g., Schneider et al. 2005). Our new analysis identifies new asymmetries, revealing that the locations of the peaks/plateaus in SB are likely shifted between the two sides, consistent with non-azimuthally symmetric structure.

4. A disk model with a flat phase function and strongly forward scattering grains where the dust ring is centered on the star reproduces the "bow" structure, marginally reproduces the "wavy" SB profile and fails to reproduce the NE/SW asymmetries. A disk model with a 5-10 AU pericenter offset reproduces the asymmetric SB profile breaks interior to $r<1^{\prime \prime}$, although its brightness asymmetry is limited to $0.5-0$ '.9 and its match to the SB at wider separations is far poorer. Thus, dust scattering plays a critical role in explaining key observed disk properties, but it is unclear whether it explains all of the disk's properties we identify.

5. HD 32297 must be surrounded by more than one dust population likely arising from different locations in the disk. Although these populations need not be identifiable from our image, we can fit the disk SED by placing dust populations at the locations of the SB peaks from our image provided that there exists additional warm dust that we cannot yet resolve.

6. The disk's brightness peak at $r \approx 0$.'4 coincides with the peak mm emission (Maness et al. 2008). If the grains responsible for both peaks are the result of collisions, then they may trace the same parent population of planetesimals.

In summary, the HD 32297 disk appearance is broadly shaped by ISM interactions (Kalas et al. 2005; Debes et al. 2009) at wide separations and by its grain scattering properties at small separations (this work). However, it is unclear whether either of these features by themselves explains the disk's SB profile and (especially) the disk's brightness asymmetry. Furthermore, SED modeling provides evidence for multiple dust populations, possibly multiple belts. To explain the disk's SB profile/multiple dust populations and (especially) brightness asymmetry, we may need to invoke other mechanisms.

Planets can sculpt dust into debris rings (e.g., Kalas 2005; Quillen et al. 2006; Kalas et al. 2008). Furthermore, planets can trap dust into resonant structures, which can appear as bright, overdense regions like the brightness peak imaged here and in the $\mathrm{mm}$ at $r \sim 0$ 0'4 (e.g., Liou \& Zook 1999; Kuchner \& Holman 2003; Stark \& Kuchner 2008). As argued by Wyatt (2006) and Maness et al. (2008), the detectability of the resonant structure may be wavelength dependent. Large grains producing $\mathrm{mm}$ emission are fragments of the colliding planetesimals in resonance, but cannot be rapidly removed by radiative forces. Thus, they can trace the parent body resonant structure. Small, (sub)micron sized grains likewise may trace resonant structure since they are preferentially produced in the most collisionally active, highest density regions and are otherwise quickly removed by radiation pressure. Grains with intermediate sizes producing emission at intermediate wavelengths (e.g., thermal IR) can be pushed out of resonance by radiation pressure/PR drag but are not small enough to be rapidly removed from the system (Wyatt 2006; though see Kuchner \& Stark 2010). Thus, it is possible that our near-IR image, when combined with the mm image, identifies planet-induced structure.

Further near-IR and (sub)mm imaging is required to verify whether the SB profiles and brightness asymmetries are bona fide evidence for an embedded planet. The current state-ofthe-art near- to mid-IR high-contrast imaging facilities like the Large Binocular Telescope have already shown great promise for resolving disks like HD 32297's in scattered light and imaging self-luminous gas giant planets (Rodigas et al. 2012; Skemer et al. 2012) and upcoming planet imagers like SCExAO on Subaru, GPI on Gemini-South, and SPHERE on the Very Large Telescope will be even more capable (Esposito et al. 2011; Martinache \& Guyon 2009; Macintosh et al. 2008; Beuzit et al. 2008). Moreover, the thermal IR is well suited for imaging exoplanets with a wide range of ages (e.g., Marois et al. 2010; Currie et al. 2011b; Rodigas et al. 2011), especially for stars like HD 32297 whose bright, edge-on debris disk degrades planet sensitivity limits in the near-IR. Imaging with the Atacama Large Millimeter Array can resolve the HD 32297 debris disk up to factor of $\sim 50$ better than the CARMA observations reported by Maness et al. (2008), and thus will provide a far better probe of any planet-induced structure in the HD 32297 debris disk.

We thank Richard Walters and Gregory Wirth for valuable help in setting up these observations, which were conducted remotely from the California Institute of Technology, and to Randy Campbell for indispensable NIRC2 support in helping the observer (T.C.) conduct his observations efficiently. Karl Stapelfeldt, Scott Kenyon, and Margaret Moerchen provided very useful discussions and the anonymous referee provided helpful comments. T.C. is supported by a NASA Postdoctoral Fellowship. The data presented herein were obtained at the W. M. Keck Observatory, which is operated as a scientific partnership among the California Institute of Technology, the University of California, and the National Aeronautics and Space Administration. The Observatory was made possible by the generous financial support of the W. M. Keck Foundation. We acknowledge the significant cultural role and reverence that the Mauna Kea summit has always had within the indigenous Hawaiian community. We are grateful to be able to conduct observations from this mountain. This research has made use of the NASA/IPAC Infrared Science Archive, which is operated by the Jet Propulsion Laboratory, California Institute of Technology, under contract with the National Aeronautics and Space Administration.

\section{APPENDIX \\ CORRECTING FOR DISK PHOTOMETRIC/ASTROMETRIC BIASES WITH LOCI}

The LOCI-based PSF subtraction approach can bias the photometry and astrometry of point sources like planets and extended structure like disks (Lafreniere et al. 2007; Thalmann et al. 2011). For point-source companions, imputing fake companions into registered images at a range of angular separations, processing these images, and then comparing the output to input fluxes and centroid positions of the fake sources corrects for these biases. Correcting biases for disks not viewed perfectly edge-on/pencil-thin is more difficult since we do not know, a priori, the disk's true FWHM, our ignorance of which also limits our ability to "debias" other disk properties whose determination depends on the disk FWHM (i.e., SB). Here, we describe our method for mitigating LOCI-based photometric/astrometric biases, which largely follows that of Rodigas et al. (2012).

We first construct model disks each with midplane brightnesses (and thus S/Ns) about twice that of the processed, real disk image and SB profiles comparable to the real disk profiles beyond $r=1^{\prime \prime}$ (Figure 10). We consider two FWHM values of $0^{\prime} .2$ and 0.25 at $1^{\prime \prime}$, or $\sim 20 \%-40 \%$ larger than the values we get between $r=0$. 3 and $1^{\prime \prime}$ prior to applying the corrections we derive in this section (Figure 11, top panels). For both runs, we 


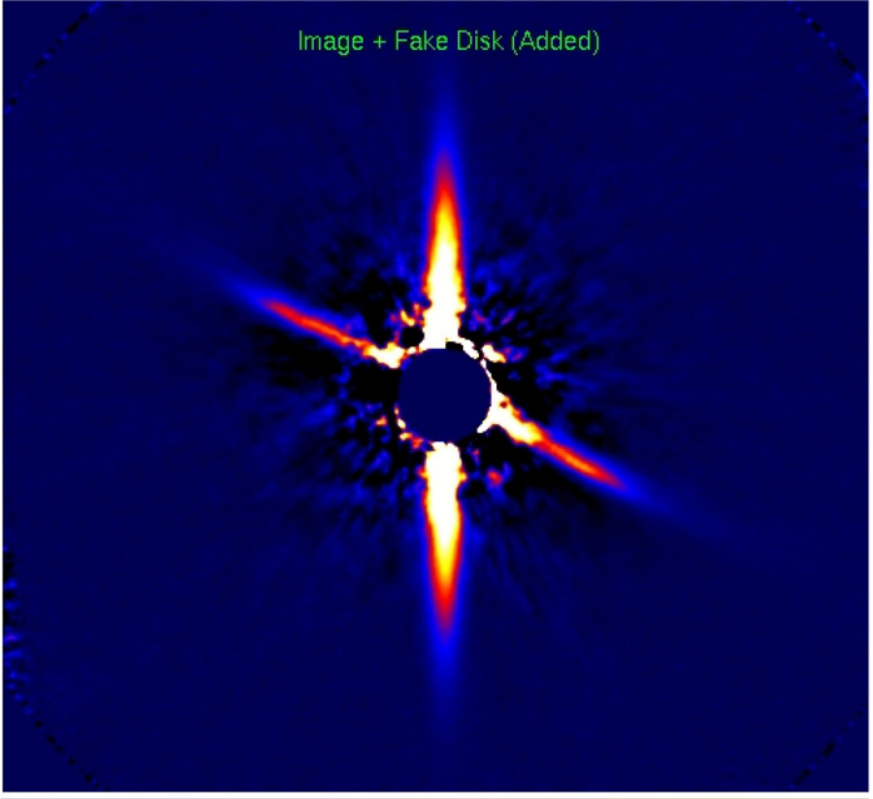

10

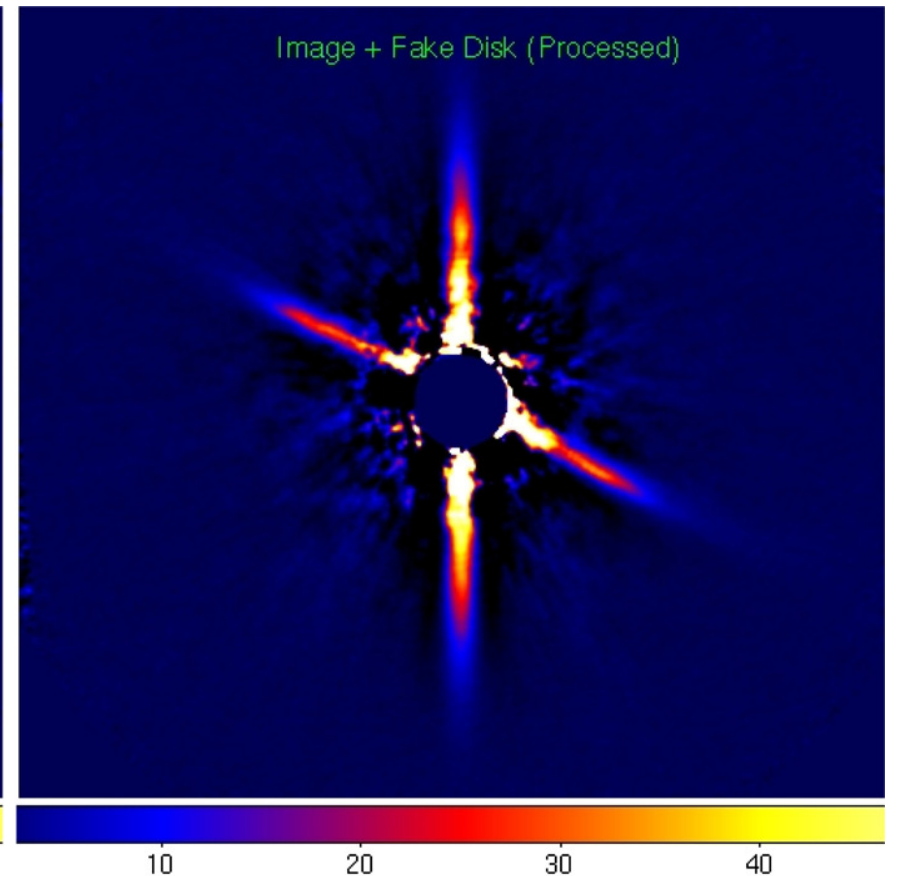

Figure 10. Images used to model biasing from LOCI processing. Left: our final image with a fake disk added. Right: our final image where we add the fake disk to each registered image and process the set of images with our pipeline. In both cases, we rotate the image to the parallactic angle of the first image in the sequence (P.A. -15.06), not to true north as we do in Figure 1.

(A color version of this figure is available in the online journal.)
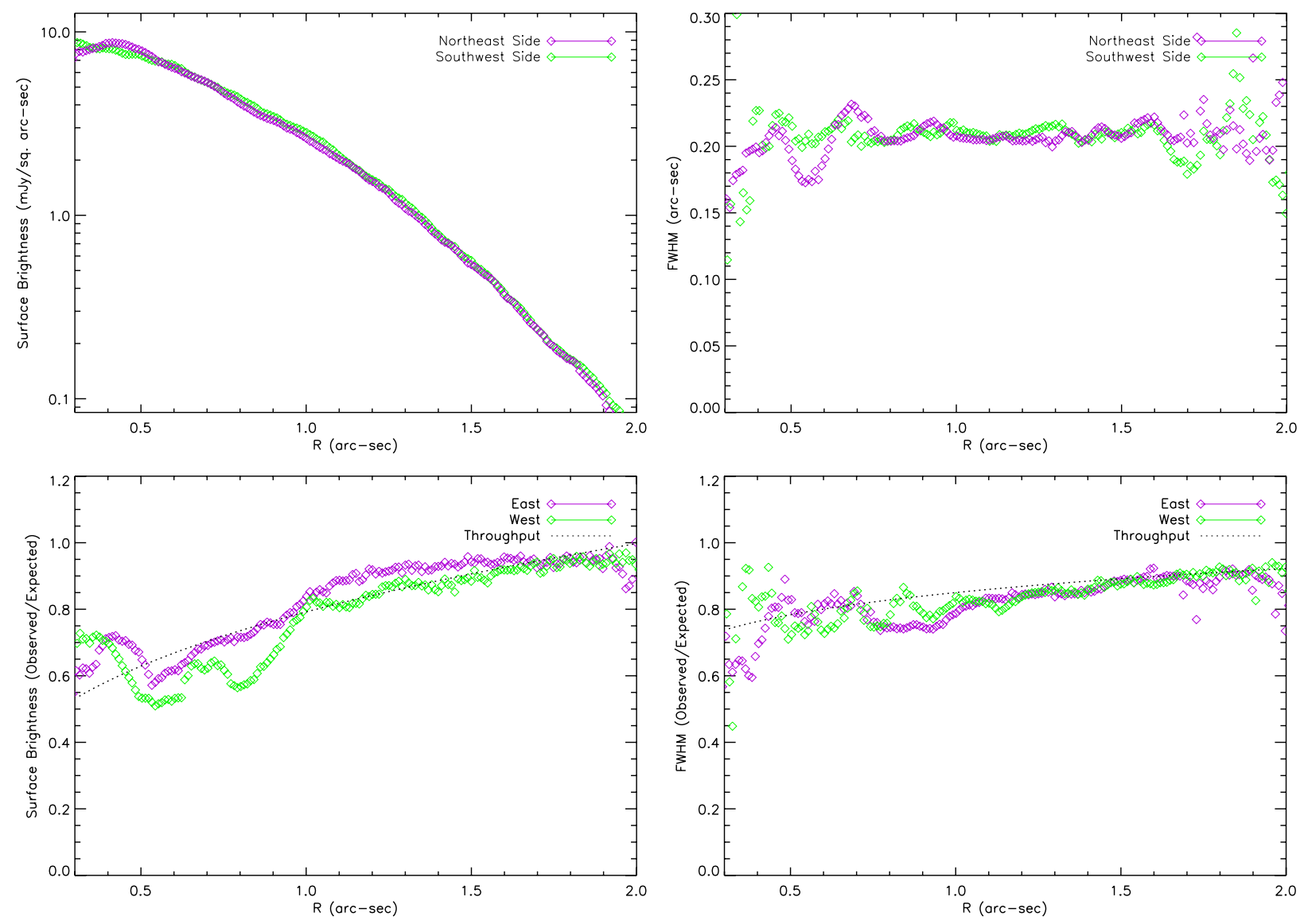

Figure 11. Top panels: surface brightness (left) and disk FWHM (right) vs. angular separation for the fake disk. Bottom panels: ratio of the "observed" (after processing) and expected surface brightness (left) and disk FWHM (right) vs. angular separation. The dotted lines identify power-law fits to correct our SB and disk FWHM measurements for biasing.

(A color version of this figure is available in the online journal.) 


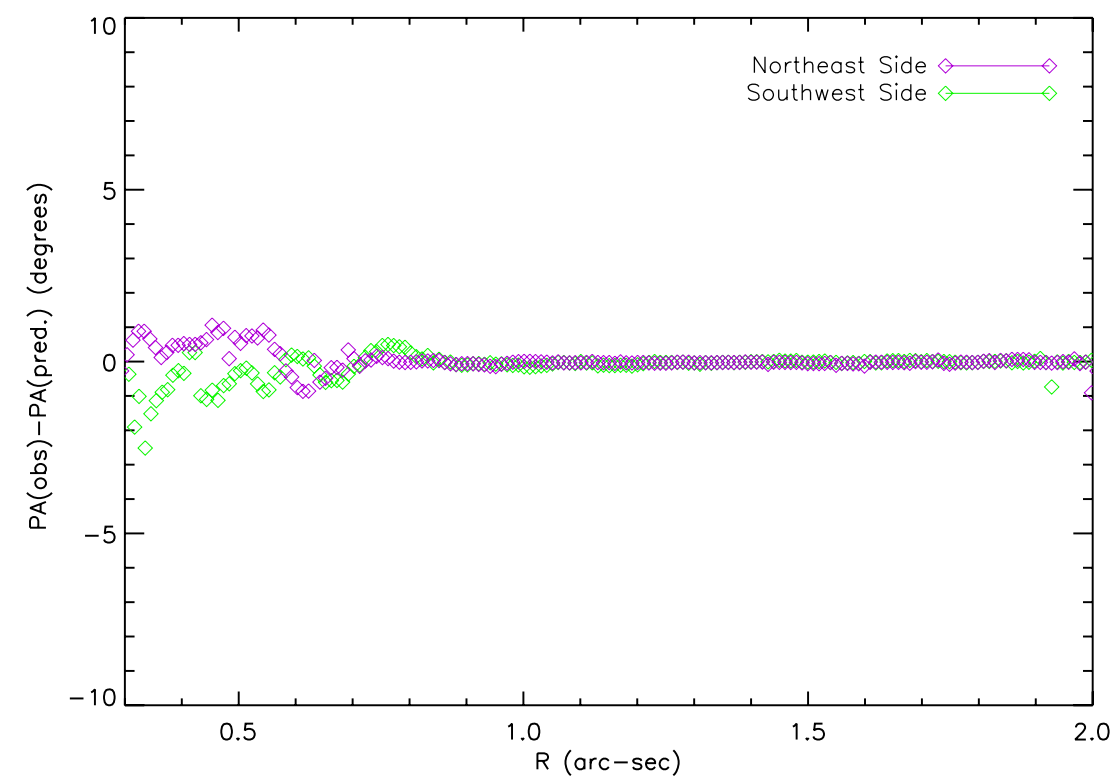

Figure 12. Difference in position angle for the fake disk before and after processing. Our processing does not bias the disk astrometry by more than $1^{\circ}-2^{\circ}$ at any separation.

(A color version of this figure is available in the online journal.)

input the fake disk into registered images, rerun our processing pipeline, and then compare the output and input SB profiles, disk thicknesses, and disk position angles.

As shown in Figure 11 (middle and bottom panels), our processing minimally biases the thinner model disk (FWHM = 0 '.2) exterior to $r=1^{\prime \prime}$, reducing its SB and thickness by no more than $20 \%$. Interior to $r=1^{\prime \prime}$, the SB and thickness drop to no less than $60 \%-75 \%$ of their original values. Biasing for the FWHM $=0$ '.25 disk (not shown) is only slightly more severe, resulting in an additional $\sim 5 \%(10 \%)$ drop at $r>1^{\prime \prime}\left(r<1^{\prime \prime}\right)$ in SB and FWHM. For both fake disks, LOCI minimally biases the disk position angle measurements (Figure 12).

The "observed" FWHM for the model with the initially thinner disk (FWHM $=0^{\prime \prime} .2$ ) is $\sim 10 \%$ smaller than the "observed FWHM for the initially thicker disk (FWHM $=0$ '.25) and more similar to that which we find for the real HD 32297 disk prior to applying any bias corrections. Thus, we derive throughput corrections for the disk SB and FWHM for the thinner disk, fitting a simple, unweighted power law to data between $r=0.3$ and $r=1^{\prime \prime} .5$.

The deviations in throughput for individual points versus our throughput function are as large as $\sim 10 \%-20 \%$. However, our modeling errors for the disk SB and FWHM are larger in regions where biasing from LOCI is important and at $r>1^{\prime \prime} .5-2^{\prime \prime}$ where the disk is intrinsically much fainter. Therefore, we leave a more detailed, robust calibration of disk parameters from LOCI-processed images to a future work where a higher $\mathrm{S} / \mathrm{N}$ disk detection at $r<1^{\prime \prime}$ will help improve our fitting precision.

\section{REFERENCES}

Augereau, J. C., Nelson, R. P., Lagrange, A. M., Papaloizou, J. C. B., \& Mouillet, D. 2001, A\&A, 370, 447

Backman, D., Witteborn, F. C., \& Gillett, F. C. 1992, ApJ, 385, 670

Beuzit, J.-L., Feldt, M., Dohlen, K., et al. 2008, Proc. SPIE, 7014, 41

Buenzli, E., Thalmann, C., Vigan, A., et al. 2010, A\&A, 524, L1

Currie, T., Bailey, V., Fabrycky, D., et al. 2010, ApJ, 721, L177

Currie, T., Burrows, A., Itoh, Y., et al. 2011a, ApJ, 729, 128

Currie, T., Fukagawa, M., Thalmann, C., Matsumura, S., \& Plavchan, P. 2012, ApJ, 755, L34
Currie, T., Kenyon, S. J., Balog, Z., et al. 2008, ApJ, 672, 558

Currie, T., Lada, C. J., Plavchan, P., et al. 2009, ApJ, 698, 1

Currie, T., Thalmann, C., Matsumura, S., et al. 2011b, ApJ, 736, L33

Dalle Ore, C. M., Fulchignoni, M., Cruikshank, D. P., et al. 2011, A\&A, 533,98

Dawson, R., Murray-Clay, R., \& Fabrycky, D. 2011, ApJ, 743, L17

Debes, J. H., Weinberger, A. J., \& Kuchner, M. J. 2009, ApJ, 702, 318

Debes, J. H., Weinberger, A. J., \& Song, I. 2008, ApJ, 684, L41

Esposito, S., Riccardi, A., Pinna, E., et al. 2011, Proc. SPIE, 8149, 1

Fitzgerald, M., Kalas, P. G., \& Graham, J. R. 2007, ApJ, 670, 557

Fitzgerald, M., Kalas, P. G., Graham, J. R., \& Maness, H. M. 2010, in Proc. Conf. on In the Spirit of Lyot 2010, Direct Detection of Exoplanets and Circumstellar Disks, 2010 October 25-29, ed. A. Boccaletti (Paris: Univ. Paris Diderot), 35

Golimowski, D., Ardila, D. R., Krist, J. E., et al. 2006, AJ, 131, 3109

Heap, S., Lindler, D. J., Lanz, T. M., et al. 2000, ApJ, 539, 435

Hines, D., Schneider, G., Hollenbach, D., et al. 2007, ApJ, 671, L165

Hong, S. S. 1985, A\&A, 146, 67

Kalas, P. 2005, ApJ, 635, L169

Kalas, P., Fitzgerald, M. P., \& Graham, J. R. 2007, ApJ, 661, L85

Kalas, P., Graham, J., \& Clampin, M. 2005, Nature, 435, 1067

Kalas, P., Graham, J. R., Chiang, E., et al. 2008, Science, 322, 1345

Kenyon, S. J., \& Bromley, B. 2008, ApJS, 179, 451

Kuchner, M., \& Holman, M. 2003, ApJ, 588, 1110

Kuchner, M., \& Stark, C. 2010, AJ, 140, 1007

Lafreniere, D., Marois, C., Doyon, R., Nadeau, D., \& Artigau, É. 2007, ApJ, 660,770

Lagrange, A.-M., Bonnefoy, M., Chauvin, G., et al. 2010, Science, 329, 57

Liou, J. C., \& Zook, H. 1999, AJ, 118, 580

Macintosh, B., Graham, J. R., Palmer, D. W., et al. 2008, Proc. SPIE, 7015, 31

Maness, H., Fitzgerald, M. P., Paladini, R., et al. 2008, ApJ, 686, L25

Marois, C., Lafrenière, D., Doyon, R., Macintosh, B., \& Nadeau, D. 2006, ApJ, 641,556

Marois, C., Macintosh, B., Barman, T., et al. 2008, Science, 322, 1348

Marois, C., Zuckerman, B., Konopacky, Q. M., Macintosh, B., \& Barman, T. 2010, Nature, 468, 1080

Martinache, F., \& Guyon, O. 2009, Proc. SPIE, 7440, 20

Mawet, D., Serabyn, E., Stapelfeldt, K., \& Crepp, J. 2009, ApJ, 702, L47

Metchev, S., Marois, C., \& Hillenbrand, L. 2009, ApJS, 181, 62

Moerchen, M., Telesco, C. M., De Buizer, J. M., Packham, C., \& Radomski, J. T. 2007, ApJ, 666, L109

Mulders, G., et al. 2012, A\&A, submitted

Murakami, H., Baba, H., Barthel, P., et al. 2007, PASJ, 59, 369

Plavchan, P., Werner, M. W., Chen, C. H., et al. 2009, ApJS, 698, 1068

Press, W., Teukolsky, S. A., Vetterling, W. T., \& Flannery, B. P. 1992, Numerical Recipes in Fortran: The Art of Scientific Computing (2nd ed.; Cambridge: Cambridge Univ. Press) 
Quillen, A. 2006, MNRAS, 372, L14

Rhee, J., Song, I., Zuckerman, B., \& McElwain, M. 2007, ApJ, 660, 1556

Rodigas, T. J., Hinz, P. M., Leisenring, J., et al. 2012, ApJ, 752, 57

Rodigas, T. J., Males, J. R., Hinz, P. M., Mamajek, E. E., \& Knox, R. P. 2011, ApJ, 732, 10

Schneider, G., Silverstone, M. D., \& Hines, D. C. 2005, ApJ, 629, L117

Skemer, A., Hinz, P. M., Esposito, S., et al. 2012, ApJ, 753, 14

Smith, B., \& Terrile, R. J. 1984, Science, 226, 1421

Stark, C., \& Kuchner, M. 2008, ApJ, 686, 637
Su, K., Rieke, G. H., Stapelfeldt, K. R., et al. 2009, ApJ, 705, 314

Thalmann, C., Janson, M., Buenzli, E., et al. 2011, ApJ, 743, L6 van Leeuwen, F. 2007, A\&A, 474, 653

Werner, M., Roellig, T. L., Low, F. J., et al. 2004, ApJS, 154, 1

Wright, E. L., Eisenhardt, P. R. M., Mainzer, A. K., et al. 2010, AJ, 140, 1868 Wyatt, M. 2006, ApJ, 639, 1153

Wyatt, M. 2008, ARA\&A, 46, 339

Wyatt, M., Dermott, S. F., Telesco, C. M., et al. 1999, ApJ, 527, 918

Yelda, S., Lu, J. R., Ghez, A. M., et al. 2010, ApJ, 725, 331 